\title{
Hilbert Schemes of Degree Four Curves
}

\author{
SCOTT NOLLET ${ }^{1}$ and ENRICO SCHLESINGER ${ }^{2}$ \\ ${ }^{1}$ Department of Mathematics, Texas Christian University, Fort Worth, TX 76129, USA. \\ e-mail:s.nollet@tcu.edu \\ ${ }^{2}$ Dipartimento di Matematica, Politecnico di Milano, 20133 Milan, Italy. \\ e-mail:enrsch@mate.polimi.it
}

(Received: 8 January 2002; accepted: 7 October 2002)

Abstract. In this paper we determine the irreducible components of the Hilbert schemes $H_{4, g}$ of locally Cohen-Macaulay space curves of degree four and arbitrary arithmetic genus $g$ : there are roughly $\sim\left(g^{2} / 24\right)$ of them, most of which are families of multiplicity structures on lines. We give deformations which show that these Hilbert schemes are connected. For $g \leqslant-3$ we exhibit a component that is disjoint from the component of extremal curves and use this to give a counterexample to a conjecture of Aït-Amrane and Perrin.

Mathematics Subject Classifications (2000). 14H50, 14C05, 14H10.

Key words. Hilbert schemes of locally Cohen-Macaulay curves, multiplicity structures on curves.

\section{Introduction}

Liaison theory has played a prominent role in classifying algebraic curves in $\mathbb{P}^{3}$ since the pioneering work of Max Noether. It has only recently become clear that locally Cohen-Macaulay curves are the natural objects of study, even if one is only interested in smooth connected curves. This is because each biliaison class is generated (via a rather explicit procedure known to the expert as the Lazarsfeld-Rao property $[4,16])$ by an essentially unique minimal curve, which need only be locally Cohen-Macaulay. For example, in the classification of smooth irreducible curves of degree 8 and genus 5 [7], one family of curves is in the biliaison class of double lines of genus -2 (non-reduced curves) and another is in the biliaison class of the disjoint unions of a line and a twisted cubic (nonconnected curves). This explains the interest in Hilbert schemes $H_{d, g}$ parametrizing locally Cohen-Macaulay curves in $\mathbb{P}^{3}$ of degree $d$ and arithmetic genus $g$.

In general, we know that the Hilbert scheme $H_{d, g}$ is non-empty precisely when $g=\frac{1}{2}(d-1)(d-2)$ (plane curves) or when $d>1$ and $g \leqslant \frac{1}{2}(d-2)(d-3)[10,3.3$ and 3.4] and that $H_{d, g}$ is reducible for $d \geqslant 3$ and $g \leqslant \frac{1}{2}(d-3)(d-4)$ with the exceptions $(d, g)=(3,0)$ and $(3,-1)[18]$. More recently it has been shown that $H_{d, g}$ is connected for $g>\left(\begin{array}{c}d-3 \\ 2\end{array}\right)-2[1,23,26]$ and that $H_{3, g}$ is connected for all $g$ [20].

The connectedness results above were obtained by deforming curves to extremal curves as introduced by Martin-Deschamps and Perrin [17]: these are the curves $C$ 
which have the largest Rao function $h^{1} \mathcal{I}_{C}(n)$ with respect to $d$ and $g$. Geometrically speaking, the extremal curves are precisely the curves of degree $d$ which contain a planar subcurve of degree $d-1$ (unless $C$ is planar or $(d, g) \in\{(3,0),(4,1)\}$ ) and they form an irreducible component $E \subset H_{d, g}$ [18]. The existence of a component of curves with the largest Rao function led Hartshorne to ask the following questions:

QUESTION 1. Is $\mathrm{H}_{\mathrm{d}, \mathrm{g}}$ connected for all $\mathrm{d}$ and $\mathrm{g}$ ?

QUESTION 2. Does each irreducible component $B \subset H_{d, g}$ meet the extremal component E?

Hartshorne showed that extremal curves can be connected to various families of curves, for example smooth rational and elliptic curves, smooth curves of degree $d \geqslant g+3$, arithmetically Cohen-Macaulay curves and many others [11]. Perrin showed that any curve with a Koszul Rao module can be deformed to an extremal curve [25]. Related to this is a conjecture of Ait-Amrane and Perrin stating that if $X$ is a family of curves whose cohomology does not exceed that of a family $X_{0}$ and the Rao module of the general curve in $X$ is a flat deformation of a subquotient of the Rao module of curves in $X_{0}$, then $\bar{X} \cap X_{0} \neq \emptyset$ : they have shown [2] that semicontinuity alone is insufficient.

Now consider curves of degree $d=4$. The Hilbert scheme $H_{4,3}$ parametrizes plane curves and is smooth irreducible of dimension $17 ; H_{4,1}$ is smooth irreducible of dimension 16 by [6] and [10,3.3 and 3.5], its general member being a complete intersection of two quadrics. The Hilbert scheme $H_{4,0}$ has two irreducible components, whose general members are respectively rational quartic curves and disjoint unions of a plane cubic and a line. Hartshorne first noticed that these two families can be connected; now there are several published proofs: $[11,13,20]$. One of our motivations is to extend the systematic study of these Hilbert schemes and complete the picture when $d=4$.

Our first theorem describes the irreducible components of $H_{4, g}$ and gives their dimensions. Our method is to first identify components whose general curve $C$ is special in the sense that $C$ is contained in a quadric surface $\left(h^{0}\left(\mathcal{I}_{C}(2)\right) \geqslant 1\right)$ or has large speciality $\left(h^{1}\left(\mathcal{O}_{C}(-1)\right) \geqslant 2\right)$. There are very few such components: if the general curve $C$ of a family lies on a quadric, then either $C$ is an extremal curve, a subextremal curve (these are the curves with largest Rao function among the nonextremal curves [21]) or a double conic. If the general curve $C$ of an irreducible component of $H_{4, g}$ has large speciality but does not lie on a quadric, we show (Proposition 4.3) that $C$ is either a thick 4-line or the union of a conic and a double line meeting at a double point. A thick 4-line [3] is a curve of degree 4 supported on a line $L$ and containing the first infinitesimal neighborhood of $L$ in $\mathbb{P}^{3}$.

Having disposed of these very special components, it is relatively easy to list those remaining. Their general member is either (a) a quasiprimitive (i.e. nonthick [3]) 
4-line, (b) the disjoint union of a line and a general curve of an irreducible component of $H_{3, g}$, or (c) the disjoint union of two double lines. Asymptotically $H_{4, g}$ has $\sim\left(g^{2} / 24\right)$ irreducible components, most of which arise from curves supported on lines. For example, from Table III we find that $H_{4,-100}$ has 530 components ( 377 of these are families of 4-lines) while $H_{4,-1,000}$ has 42755 components (of which 41252 arise from 4-lines).

Our second theorem states that $H_{4, g}$ is connected when it is not empty. The main novelty for $g \leqslant-3$ is the presence of a component $G_{4}$ consisting entirely of thick 4 -lines. We prove the connectedness theorem by showing that each irreducible component can be connected either to the extremal component $E$ or to $G_{4}$, and that the component of subextremal curves meets both $E$ and $G_{4}$. Specifically, the quasi-primitive 4-lines and the curves with large speciality deform to thick 4-lines (Sections 2 and 4). We show that a disjoint union of double lines specializes to a quasi-primitive 4-line on a double quadric surface in Section 3. Section 5 is devoted to showing that families of unions of triple lines and reduced lines can be connected to the extremal component.

The component $G_{4}$ of thick 4-lines turns out to be rather interesting. Since these curves are scheme-theoretically (although not cohomologically) the most special, they cannot specialize to extremal curves, answering Question 2 in the negative. Since their Rao modules are flat deformations of subquotients of the Rao modules of extremal curves (Example 6.7), we obtain a counterexample to the conjecture of Ait-Amrane and Perrin above. Question 1 remains open.

Throughout the paper we work over an algebraically closed field $k$ of arbitrary characteristic. A curve for us is a locally Cohen-Macaulay scheme over $k$ of pure dimension 1 . We will freely use the sentence 'the family of curves of degree $d$ and genus $g$ with property $P$ is irreducible of dimension $m$ ' to mean that there is a (unique) irreducible $m$-dimensional constructible subset $S$ of the Hilbert scheme $H_{d, g}$ whose closed points parametrize the curves of degree $d$ and genus $g$ with property $P$. Note that, since $S$ is constructible, the closure of $S$ in the Hilbert scheme is also an $m$-dimensional irreducible subset.

\section{Multiplicity Four Structures on Lines}

In this section we study locally Cohen-Macaulay curves in $\mathrm{P}^{3}$ which are supported on a line; we will simply call these $d$-lines, where $d$ is the degree of the curve.

We begin with the general theory of Banica and Forster [3, $\S 3]$. Let $C$ be a locally Cohen-Macaulay curve on a smooth threefold $X$ with smooth support $Y$. Letting $Y^{(i)}$ be the subscheme of $X$ defined by $\mathcal{I}_{Y}^{i}$ and $C_{i}$ be the subscheme of $X$ obtained by removing the embedded points from $C \cap Y^{(i)}$, we obtain the Cohen-Macaulay filtration for $C$ :

$$
Y=C_{1} \subset C_{2} \subset \cdots \subset C_{k}=C
$$


for some $k \geqslant 1$. The quotients $L_{j}=\mathcal{I}_{C_{j}} / \mathcal{I}_{C_{j+1}}$ are vector bundles on $Y$ and the multiplicity is given by $\mu(C)=1+\sum \operatorname{rank} L_{j}$. The natural inclusions $\mathcal{I}_{C_{i}} \mathcal{I}_{C_{j}} \subset \mathcal{I}_{C_{i+j}}$ induce generically surjective multiplication maps $L_{i} \otimes L_{j} \rightarrow L_{i+j}$ and in particular we obtain generic surjections $L_{1}^{j} \rightarrow L_{j}$.

As in [3, 4], the curve $C$ is said to be thick if $Y^{(2)} \subset C$; this is equivalent to the condition that $C$ has embedding dimension three at each point. In terms of filtration (1) above, this says that $C_{2}=Y^{(2)}$, in which case $L_{1}=\mathcal{I}_{Y} / \mathcal{I}_{Y}^{2}$ is the conormal bundle of $Y$ on $X$. If further $\mu(C)=4$, then rank $L_{2}=1$ and there is an exact sequence

$$
0 \rightarrow \frac{\mathcal{I}_{C}}{\mathcal{I}_{Y}^{3}} \rightarrow \frac{\mathcal{I}_{Y}^{2}}{\mathcal{I}_{Y}^{3}} \rightarrow L_{2} \longrightarrow 0
$$

For a line $Y \subset \mathbb{P}^{3}$, we obtain the following.

PROPOSITION 2.1. For $g \leqslant 1$, the thick 4-lines in $H_{4, g}$ form an irreducible closed subset of dimension $9-3 g$.

Proof. The condition that $C$ contain $Y^{(2)}$ is clearly closed. If $C$ is a thick 4-line with support $Y \subset \mathbb{P}^{3}$, then $\mu(C)=4, \mathcal{I}_{Y}^{2} / \mathcal{I}_{Y}^{3} \cong \mathcal{O}_{Y}(-2)^{\oplus 3}$ and $L_{2} \cong \mathcal{O}_{Y}(-g-1)$ (because Pic $Y \cong Z$ ). It follows from sequence (2) above that giving such a curve $C$ is the same as giving a surjective morphism $\mathcal{O}_{Y}(-2)^{\oplus 3} \rightarrow \mathcal{O}_{Y}(-g-1)$ modulo an automorphism of $\mathcal{O}_{Y}(-g-1)$. Thus the set of thick 4-lines of genus $g$ is parametrized by an open subset of a $\mathbb{P}^{5-3 g}$-bundle over the Hilbert scheme of lines in $\mathbb{P}^{3}$.

On the other hand, a curve $C$ is quasiprimitive if it is not thick, which is equivalent to having generic embedding dimension two. In this case rank $L_{1}=1$ and the generic surjections $L_{1}^{j} \rightarrow L_{j}$ yield effective divisors $D_{j}$ such that $L_{j} \cong L_{1}^{j}\left(D_{j}\right)$; the multiplication maps show that $D_{i}+D_{j} \leqslant D_{i+j}$. If $C$ is a quasiprimitive 4-line in $\mathrm{P}^{3}$, then the Cohen-Macaulay filtration takes the form $Y \subset D \subset W \subset C$ where $Y$ is a line, $D$ a double line and $W$ a triple line. Setting $a=\operatorname{deg} L_{1}, b=\operatorname{deg} D_{2}$ and $c=\operatorname{deg} D_{3}$, the triple $(a, b, c)$ is the type of $C$. Since the surjection $\mathcal{I}_{Y} \rightarrow \mathcal{O}_{Y}(a)$ factors through the conormal bundle $\mathcal{I}_{Y} / \mathcal{I}_{Y}^{2} \cong \mathcal{O}_{Y}(-1)^{2}$, we necessarily have $a \geqslant-1$, while by construction $b, c \geqslant 0$. That $C$ has arithmetic genus $p_{a}(C)=-6 a-b-$ $c-3$ follows from the isomorphisms $\mathcal{I}_{Y, D} \cong \mathcal{O}_{Y}(a), \mathcal{I}_{D, W} \cong \mathcal{O}_{Y}(2 a+b)$ and $\mathcal{I}_{W, C} \cong \mathcal{O}_{Y}(3 a+c)$.

The following lemma helps us construct 4-lines from quasiprimitive 3-lines.

LEMMA 2.2. Let $W \subset \mathbb{P}^{3}$ be a quasiprimitive 3-line of type $(a, b, c)$ with support $Y$ and underlying double line $D$. Set $\mathcal{J}=\mathcal{I}_{W} \mathcal{I}_{Y}+\mathcal{I}_{D}^{2}$. Then in the short exact sequence

$$
0 \longrightarrow \frac{\mathcal{I}_{Y} \mathcal{I}_{D}}{\mathcal{J}} \longrightarrow \frac{\mathcal{I}_{W}}{\mathcal{J}} \rightarrow \frac{\mathcal{I}_{W}}{\mathcal{I}_{Y} \mathcal{I}_{D}} \longrightarrow 0
$$

we have isomorphisms

$$
\frac{\mathcal{I}_{Y} \mathcal{I}_{D}}{\mathcal{J}} \cong \mathcal{O}_{Y}(3 a+b) \text { and } \quad \frac{\mathcal{I}_{W}}{\mathcal{I}_{Y} \mathcal{I}_{D}} \cong \mathcal{O}_{Y}(-a-b-2) .
$$


In particular, when $a \geqslant 0$, the sequence is split exact.

Proof. On the open set where $W$ has embedding dimension two, the quotient $\mathcal{I}_{Y} \mathcal{I}_{D} / \mathcal{J}$ is an invertible $\mathcal{O}_{Y}$-module. It follows that the multiplication map $\mathcal{I}_{Y, D} \otimes_{\mathcal{O}_{Y}} \mathcal{I}_{D, W} \stackrel{\mu}{\rightarrow} \mathcal{I}_{Y} \mathcal{I}_{D} / \mathcal{J}$ is an isomorphism (it is surjective a priori; if $\operatorname{ker} \mu \neq 0$, then the image has finite support) and $\mathcal{I}_{Y} \mathcal{I}_{D} / \mathcal{J} \cong \mathcal{O}_{Y}(3 a+b)$. Further, $\mathcal{I}_{D} / \mathcal{I}_{Y} \mathcal{I}_{D} \cong$ $\mathcal{O}_{Y}(2 a) \oplus \mathcal{O}_{Y}(-a-2)$ by $[20,2.3]$ and the exact sequence

$$
0 \rightarrow \mathcal{I}_{W} / \mathcal{I}_{Y} \mathcal{I}_{D} \rightarrow \mathcal{I}_{D} / \mathcal{I}_{Y} \mathcal{I}_{D} \rightarrow \mathcal{O}_{Y}(2 a+b) \rightarrow 0
$$

shows that $\mathcal{I}_{W} / \mathcal{I}_{Y} \mathcal{I}_{D} \cong \mathcal{O}_{Y}(-a-b-2)$. When $a \geqslant 0$, the sequence must split because

$$
\operatorname{Ext}_{Y}^{1}\left(\mathcal{O}_{Y}(-a-b-2), \mathcal{O}_{Y}(3 a+b)\right) \cong \mathrm{H}^{1}\left(Y, \mathcal{O}_{Y}(4 a+2 b+2)\right)=0 .
$$

If a quasiprimitive 4-line $C$ has type $(a, b, c)$ with $a=-1$, then $C$ necessarily lies in a double plane and, hence, is a flat limit of double conics [14, 8.1 and 8.2]. Since we are mainly interested in families that form irreducible components of the Hilbert scheme, we will assume that $a \geqslant 0$ in the sequel. The following proposition is based on $[3, \S 3.8]$.

PROPOSITION 2.3. Let $(a, b, c)$ be a triple of integers satisfying $a \geqslant 0$ and $0 \leqslant b \leqslant c$. Then the quasiprimitive 4-lines of type $(a, b, c)$ in $H_{4, g}$ form an irreducible constructible subset of dimension $9 a+2 b+2 c+13$.

Proof. The set of double lines of type $a$ is parametrized by an open subscheme $V_{2}$ of a $\mathbb{P}^{2 a+3}$-bundle over the Grassmannian of lines in $\mathbb{P}^{3}[20,1.6]$. Indeed, to give a double structure $D$ of type $a$ on a line $Y$ is equivalent to give a surjective morphism $\mathcal{I}_{Y} / \mathcal{I}_{Y}^{2} \cong \mathcal{O}_{Y}(-1)^{2} \stackrel{[f g]}{\rightarrow} \mathcal{O}_{Y}(a)$ modulo an automorphism of $\mathcal{O}_{Y}(a)$.

Similarly, the quasiprimitive triple lines of type $(a, b)$ which contain a double line $D \in V_{2}$ are determined by surjective morphisms $\mathcal{I}_{D} / \mathcal{I}_{Y} \mathcal{I} D \cong \mathcal{O}_{Y}(2 a) \oplus \mathcal{O}_{Y}(-a-2)$ $\stackrel{[p q]}{\rightarrow} \mathcal{O}_{Y}(2 a+b)$ modulo automorphisms of $\mathcal{O}_{Y}(2 a+b)$, the isomorphism $\tau$ being given in $[20,2.3]$. It follows that the set of triple lines of type $(a, b)$ is parametrized by an open subscheme $V_{3}$ of a $\mathrm{P}^{3 a+2 b+3}$-bundle over $V_{2}$, hence is irreducible of dimension $5 a+2 b+10$.

In the same way, the 4-lines of type $(a, b, c)$ which contain a fixed quasiprimitive 3-line $W$ of type $(a, b)$ with support $Y$ are determined by surjections $\mathcal{I}_{W} / \mathcal{I}_{Y} \mathcal{I}_{W} \stackrel{\phi}{\rightarrow}$ $\mathcal{O}_{Y}(3 a+c)$. Since $\mathcal{I}_{D}^{2} \subset \mathcal{I}_{Y} \mathcal{I}_{W}$ on an open set, the image of $\mathcal{I}_{D}^{2}$ in $\mathcal{I}_{W} / \mathcal{I}_{Y} \mathcal{I}_{W}$ is torsion so that $\phi$ factors through $\mathcal{I}_{W} / \mathcal{J}$, where $\mathcal{J}=\mathcal{I}_{Y} \mathcal{I}_{W}+\mathcal{I}_{D}^{2}$, allowing us to use Lemma 2.2. Thus the surjections $\bar{\phi}: \mathcal{I}_{W} / \mathcal{J} \cong \mathcal{O}_{Y}(3 a+b) \oplus \mathcal{O}_{Y}(-a-b-2) \stackrel{[r s]}{\rightarrow} \mathcal{O}_{Y}(3 a+c)$ modulo automorphisms of $\mathcal{O}_{Y}(3 a+c)$ are parametrized by an open subset $V_{4}$ of a $\mathrm{P}^{4 a+2 c+3}$-bundle over $V_{3}$, that is, by an irreducible variety of dimension $9 a+2 b+$ $2 c+13$.

We now show that each surjection $\bar{\phi}$ gives a quasiprimitive 4-line. Since $W$ is quasiprimitive, there are local coordinates $s$ and $t$ on an open set with $I_{Y}=(s, t)$, $I_{D}=\left(s, t^{2}\right)$, and $I_{W}=\left(s, t^{3}\right)[3,2.1]$. In particular, $(s, t)^{3}=I_{Y}^{3} \not \subset \mathcal{J}=\left(s^{2}, s t, t^{3}\right)$ so that the image of $\mathcal{I}_{Y}^{3}$ is nonzero in $\mathcal{I}_{Y} \mathcal{I}_{D} / \mathcal{J} \cong \mathcal{O}_{Y}(3 a+b)$ and for any surjection $\phi$, 
the induced map $\mathcal{O}_{Y}(3 a+b) \rightarrow \mathcal{O}_{Y}(3 a+c)$ is injective, whence the image of $\mathcal{I}_{Y}^{3}$ is nonzero in $\mathcal{O}_{Y}(3 a+c)$ and so $C \not \subset Y^{(3)}$ : from this it follows that $C$ is quasiprimitive via the Cohen-Macaulay filtration.

The following result is an important ingredient in our connectedness theorem 6.4.

PROPOSITION 2.4. Let $(a, b, c)$ be $a$ triple with $a \geqslant 0$ and $c \geqslant b \geqslant 0$. Then there exists a flat family of curves $C \subset \mathbb{P}_{k[t]}^{3}$ such that

(1) the fibre $C_{t}$ is a quasiprimitive 4-line of type $(a, b, c)$ for $t \neq 0$

(2) the fibre $C_{0}$ is a thick 4-line.

Proof. The outline of the proof is as follows. Fixing a double line $Z$ of type (a) supported on the line $L$, we have seen above that a quasiprimitive triple line of type $(a, b)$ containing $Z$ is determined by a surjective morphism $\mathcal{I}_{Z} \stackrel{\psi}{\rightarrow} \mathcal{O}_{L}(2 a+b)$. We construct a family of such surjections $\psi_{t}$ for $t \neq 0$ whose limit at $t=0$ is a morphism $\psi_{0}: \mathcal{I}_{Z} \rightarrow \mathcal{O}_{L}(-a-2) \oplus \mathcal{O}_{D}$ where $D$ is an effective divisor on $L$. Then ker $\psi_{t}$ defines a family $W_{t}$ whose general fibre is a quasiprimitive 3-line of type $(a, b)$, while the special fibre $W_{0}$ is the first infinitesimal neighborhood of $L$ plus some embedded points along $D$. We construct the desired family $C_{t}$ by picking a morphism $\mathcal{I}_{W_{t}} \rightarrow \mathcal{O}_{L}(3 a+c)$ in such a way that the 'extra line' in $C_{0}$ covers the embedded points of $W_{0}$, so that $C_{0}$ is locally Cohen-Macaulay.

Fix coordinates so that $\mathbb{P}^{3}=\operatorname{Proj}(k[x, y, z, w])$ and let $L_{0}$ be the line with equations $x=y=0$. Let $Z_{0}$ be the double structure on $L_{0}$ defined by the homogeneous ideal $\left(x^{2}, x y, y^{2}, x g-y f\right)$ where $f=z^{a+1}$ and $g=w^{a+1}$, thus $Z_{0}$ is a double line of genus $-a-1$. Now consider the trivial families $L=L_{0} \times \mathrm{A}^{1}$ and $Z=Z_{0} \times \mathrm{A}^{1}$ over $\mathrm{A}^{1}=\operatorname{Spec} k[t]$ and let $\mathcal{I}_{Z}$ denote the ideal sheaf of $Z$ in $\mathrm{P}^{3} \times \mathrm{A}^{1}$. Then we have an isomorphism $\mathcal{I}_{Z} \otimes \mathcal{O}_{L} \cong \mathcal{O}_{L}(-a-2) \oplus \mathcal{O}_{L}(2 a)$ in which the natural epimorphism $\pi: \mathcal{I}_{Z} \rightarrow \mathcal{I}_{Z} \otimes \mathcal{O}_{L} \cong \mathcal{O}_{L}(-a-2) \oplus \mathcal{O}_{L}(2 a)$ sends $x g-y f$ to $(1,0)$ and $x^{2}, x y$ and $y^{2}$ to $\left(0, f^{2}\right),(0, f g),\left(0, g^{2}\right)[20,2.3]$.

Consider the injective morphism

$$
\chi: \mathcal{O}_{L}(-a-b-2) \stackrel{\left[\begin{array}{c}
t w^{b} \\
z^{3 a+b+2}
\end{array}\right]}{\longrightarrow} \mathcal{O}_{L}(-a-2) \oplus \mathcal{O}_{L}(2 a)
$$

and set $\mathcal{G}=\operatorname{coker}(\chi)$. Note that $\mathcal{G}$ is flat over $\mathrm{A}^{1}$ because $\chi$ remains injective on the fibres over $\mathrm{A}^{1}$. Now let $\psi: \mathcal{I}_{Z} \rightarrow \mathcal{G}$ be the composite surjection

$$
\mathcal{I}_{Z} \stackrel{\pi}{\rightarrow} \mathcal{O}_{L}(-a-2) \oplus \mathcal{O}_{L}(2 a) \rightarrow \mathcal{G}
$$

and define $W$ by $\mathcal{I}_{W}=\operatorname{ker}(\psi)$.

It is clear that $W$ is a flat family of closed subschemes over $A^{1}$. For $t \neq 0$, $\mathcal{G}_{t}=\mathcal{G} \otimes k(t)$ is isomorphic to $\mathcal{O}_{L_{0}}(2 a+b)$ so that $W_{t}$ is a quasiprimitive triple line of type $(a, b)$. On the other hand, $\mathcal{G}_{0}$ is isomorphic to $\mathcal{O}_{L_{0}}(-a-2) \oplus \mathcal{O}_{D}$, where $D$ is the divisor in $L_{0}$ defined by the global section $s=z^{3 a+b+2}$ of $\mathcal{O}_{L_{0}}(3 a+b+2)$. By construction $W_{0}$ contains $L_{0}^{(2)}$ and $\mathcal{I}_{L_{0}^{(2)}, W_{0}} \cong \mathcal{O}_{D}$. 
We now claim that, over the coordinate ring $R=k[t][x, y, z, w]$ of $\mathbb{P}_{k[t]}^{3}$, the saturated ideal $I_{W}$ of $W \subset \mathbb{P}_{k[t]}^{3}$ has a free graded resolution

$$
0 \rightarrow F_{3} \stackrel{M_{3}}{\rightarrow} F_{2} \stackrel{M_{2}}{\rightarrow} F_{1} \stackrel{M_{1}}{\rightarrow} F_{0}=R,
$$

where

$$
\begin{aligned}
& F_{1}=R(-3)^{\oplus 4} \oplus R(-a-3)^{\oplus 2} \oplus R(-a-b-2), \\
& F_{2}=R(-4)^{\oplus 3} \oplus R(-a-4)^{\oplus 4} \oplus R(-a-b-3)^{\oplus 2}, \\
& F_{3}=R(-a-5)^{\oplus 2} \oplus R(-a-b-2),
\end{aligned}
$$

and the maps are defined by the matrix transposes

$$
\begin{gathered}
M_{1}^{t}=\left[\begin{array}{c}
x^{3} \\
x^{2} y \\
x y^{2} \\
y^{3} \\
x(x g-y f) \\
y(x g-y f) \\
x^{2} z^{a+b}+t w^{b}(x g-y f)
\end{array}\right], \quad M_{3}^{t}=\left[\begin{array}{ccc}
g & 0 & z^{a+b} \\
-f & g & 0 \\
0 & -f & 0 \\
y & 0 & 0 \\
-x & y & 0 \\
0 & -x & 0 \\
0 & y & -t w^{b} \\
0 & 0 & -y \\
0 & 0 & x
\end{array}\right], \\
\left.M_{2}^{t}=\left[\begin{array}{ccccccc}
y & -x & 0 & 0 & 0 & 0 & 0 \\
0 & y & -x & 0 & 0 & 0 & 0 \\
0 & 0 & y & -x & 0 & 0 & 0 \\
-g & f & 0 & 0 & x & 0 & 0 \\
0 & -g & f & 0 & y & 0 & 0 \\
0 & 0 & -g & f & 0 & y & 0 \\
0 & 0 & 0 & 0 & -y & x & 0 \\
z^{a+b} & 0 & 0 & 0 & t w^{b} & 0 & -x \\
0 & z^{a+b} & 0 & 0 & 0 & t w^{b} & -y
\end{array}\right], \quad \begin{array}{c}
-t f^{3} z^{c-b} w^{b} \\
-t f^{2} g z^{c-b} w^{b} \\
-t f g^{2} z^{c-b} w^{b} \\
-t g^{3} z^{c-b} w^{b} \\
z^{4 a+c+3} \\
z^{3 a+c+2} w^{a+1} \\
w^{4 a+b+c+2}
\end{array}\right] .
\end{gathered}
$$

(In the second line we have included a matrix $P$ which will be used a few lines below).

We use the Buchsbaum-Eisenbud criterion [5] to see that the complex above is acyclic. For this it is enough to observe that the ideal $I_{3}\left(M_{3}\right)$ generated by the $3 \times 3$ minors of $M_{3}$ contains the regular sequence $\left(x^{3}, y^{3}, z^{3 a+b+2}\right)$ and that $I_{6}\left(M_{2}\right)$ contains the regular sequence $\left(x^{6}, y^{6}\right)$. Therefore the complex is a resolution of the ideal $J$ defined by $M_{1}$, hence $J$ is the saturated homogeneous ideal of a closed subscheme of $\mathbb{P}_{k[t]}^{3}$. It is clear that $J \subseteq I_{W}$, and comparing the Hilbert polynomials we see $J=I_{W}$. This proves the claim.

We now construct a family $C$ of 4-lines as in the statement of the Proposition. Recalling that $f=w^{a+1}$ and $g=z^{a+1}$, one checks that the map $F_{1} \stackrel{P}{\rightarrow} R(3 a+c)$ satisfies $P M_{2}=0 \bmod I_{L}$, where $I_{L}=(x, y)$ is the ideal of $L$ (here $P$ is the matrix above). We thus obtain a map $I_{W} \stackrel{\bar{P}}{\rightarrow} T(3 a+c)$ where $T=R / I_{L} \cong A[z, w]$. Sheafifying, we obtain a morphism $\phi: \mathcal{I}_{W} \rightarrow \mathcal{O}_{L}(3 a+c)$ and $\mathcal{I}_{C}=\operatorname{ker} \phi$ defines $C$. Since $\phi_{t}$ is 
surjective for all $t \in \mathrm{A}^{1}$, we see from the proof of Proposition 2.3 above that $C_{t}$ is a quasiprimitive 4-line of type $(a, b, c)$ for $t \neq 0$.

Finally, we check that $C_{0}$ is a thick 4-line. To this end, observe that

$$
\mathcal{I}_{L_{0}}^{3} \subset \mathcal{I}_{W_{0}} \subset \mathcal{I}_{L_{0}}^{2}
$$

and that $\phi_{0}$ vanishes on $\mathcal{I}_{L_{0}}^{3}$, hence factors through $\mathcal{I}_{W_{0}} / \mathcal{I}_{L_{0}}^{3}$. On the other hand, looking at the presentation of $I_{W} \otimes T$, we see that $\mathcal{I}_{W_{0}} / \mathcal{I}_{L_{0}}^{3}$ is isomorphic to

$$
\mathcal{O}_{L_{0}}(-a-3)^{\oplus 2} \oplus \mathcal{O}_{L_{0}}(-a-b-2) .
$$

Thus we have a commutative diagram of short exact sequences:

$$
\begin{aligned}
& 0 \rightarrow \begin{array}{c}
\mathcal{I}_{C_{0}} / \mathcal{I}_{L_{0}}^{3} \\
\downarrow
\end{array} \rightarrow \begin{array}{cccc}
\mathcal{I}_{W_{0}} / \mathcal{I}_{L_{0}}^{3} & \stackrel{\phi_{0}}{\rightarrow} & \mathcal{O}_{L_{0}}(3 a+c) \\
\downarrow & \downarrow^{3 a+b+2}
\end{array} \quad \rightarrow 0 \\
& 0 \rightarrow \mathcal{I}_{\bar{C}} / \mathcal{I}_{L_{0}}^{3} \rightarrow \mathcal{I}_{L_{0}}^{2} / \mathcal{I}_{L_{0}}^{3} \stackrel{\beta}{\rightarrow} \mathcal{O}_{L_{0}}(6 a+b+c+2) \rightarrow 0
\end{aligned}
$$

where, identifying $\mathcal{I}_{W_{0}} / \mathcal{I}_{L_{0}}^{3}$ with $\mathcal{O}_{L_{0}}(-a-3)^{\oplus 2} \oplus \mathcal{O}_{L_{0}}(-a-b-2)$ and $\mathcal{I}_{L_{0}}^{2} / \mathcal{I}_{L_{0}}^{3}$ with $\mathcal{O}_{L_{0}}(-2)^{\oplus 3}$, the morphisms are

$$
\begin{aligned}
& \alpha=\left[\begin{array}{ccc}
w^{a+1} & 0 & z^{a+b} \\
-z^{a+1} & w^{a+1} & 0 \\
0 & -z^{a+1} & 0
\end{array}\right], \quad \beta^{t}=\left[\begin{array}{c}
z^{2 a+2} w^{4 a+b+c+2} \\
z^{a+1} w^{5 a+b+c+3}-z^{6 a+b+c+4} \\
w^{6 a+b+c+4}-2 z^{5 a+b+c+3} w^{a+1}
\end{array}\right], \\
& \bar{\phi}_{0}=\left[\begin{array}{lll}
z^{4 a+c+3} & z^{3 a+c+2} w^{a+1} & w^{4 a+b+c+2}
\end{array}\right] .
\end{aligned}
$$

Since $\alpha$ is injective and

$$
\operatorname{deg}\left(\mathcal{I}_{L_{0}}^{2} / \mathcal{I}_{L_{0}}^{3}\right)-\operatorname{deg}\left(\mathcal{I}_{W_{0}} / \mathcal{I}_{L_{0}}^{3}\right)=3 a+b+2,
$$

we see that $C_{0}=\bar{C}$ is a thick 4-line, and this concludes the proof.

Remark 2.5. We don't know what kind of specializations might occur between quasiprimitive four-lines in general, but we formulate the following necessary condition: if a family of quasiprimitive four-lines of type $(a, b, c)$ specializes to a four-line of type $\left(a^{\prime}, b^{\prime}, c^{\prime}\right)$, then $a^{\prime} \leqslant a$. To see this, consider the deformation of the underlying double line $Z$ : the general such $Z$ has genus $-1-a$, hence the limit $Z^{\prime}$ consists of a double line of genus $-1-a^{\prime}$ and possibly some embedded points. Since the arithmetic genus is constant, we conclude that $a^{\prime} \leqslant a$. Similarly, if a family of triple lines of type $(a, b)$ specializes to another of type $\left(a^{\prime}, b^{\prime}\right)$, then $a^{\prime} \leqslant a$. Examples of both types of specialization are seen in [20, 3.6 and 3.10].

\section{A Deformation on the Double Quadric}

In this section we apply results from our general study of curves on double surfaces [24] to show that a disjoint union of double lines specializes to certain quasiprimitive 4-lines on a double quadric. 
Let $Q \subset \mathbb{P}^{3}$ be a smooth quadric surface and let $X \subset \mathbb{P}^{3}$ be the effective divisor $2 Q$. For a curve $C \subset X$, let $P$ be the curve part of the scheme-theoretic intersection $C \cap Q$. We may write

$$
\mathcal{I}_{C \cap Q, Q}=\mathcal{I}_{Z, Q}(-P)
$$

where $Z$ is zero-dimensional. The inclusion $P \subset C \cap Q$ generates the commutative diagram

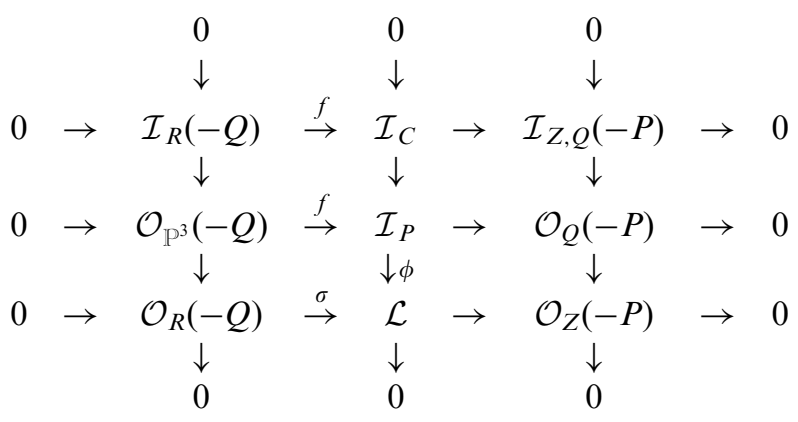

in which $f$ is the equation for $Q \subset \mathbb{P}^{3}$. This diagram defines the residual curve $R$ to $C$ in $Q$ and we obtain a triple $T(C)=\{Z, R, P\}$ in which $R \subseteq P$ are effective divisors on $Q$. Using depth arguments and results on generalized divisors [9] as in [14], $Z$ is a Gorenstein divisor on $R, \mathcal{L} \cong \mathcal{O}_{R}(Z-Q)$ is a rank one reflexive $\mathcal{O}_{R}$-module, and $\sigma$ gives the section of $\mathcal{L}(Q)$ defining $Z$ as a generalized divisor on $R[24, \S 2]$. The arithmetic genus of $C$ is given by the formula

$$
g(C)=g(P)+g(R)+\operatorname{deg}_{R} \mathcal{O}_{R}(Q)-\operatorname{deg}(Z)-1 .
$$

PROPOSITION 3.1. Let $C \subset P^{3}$ be a general quasiprimitive 4-line of type $(0, b, c)$ with Cohen-Macaulay filtration $L \subset D \subset W \subset C$. Then there exists a smooth quadric surface $Q \subset \mathbb{P}^{3}$ such that

(1) $D \subset Q$ and $C \subset 2 Q$.

(2) The triple associated to $C \subset 2 Q$ has form $T(C)=\{Z, D, D\}$, where $Z$ consists of $c-b$ simple points and $b+2$ double points, none of which is contained in $L$.

(3) $H^{1}\left(\mathcal{O}_{D}(Z+D-Q)\right)=0$.

Proof. That $D$ is contained in a smooth quadric $Q$ is [20, 1.5], hence $\mathcal{I}_{2 Q} \subset \mathcal{I}_{D}^{2} \subset$ $\mathcal{I}_{C}$ and $C \subset 2 Q$. The definition of type of $C$ gives rise to the top row in the diagram:

$$
\begin{aligned}
& 0 \rightarrow \mathcal{I}_{W} \rightarrow \begin{array}{l}
\mathcal{I}_{D} \\
\pi \downarrow
\end{array} \stackrel{\psi}{\rightarrow} \quad \begin{array}{l}
\mathcal{O}_{L}(b) \\
{[p q] \uparrow}
\end{array} \rightarrow 0 \\
& \mathcal{I}_{D} \otimes \mathcal{O}_{L} \stackrel{\sim}{\rightarrow} \mathcal{O}_{L} \oplus \mathcal{O}_{L}(-2)
\end{aligned}
$$

in which $\psi$ factors through $\pi$ and the isomorphism along the bottom row takes the equation $f$ for $Q$ to the generator of the summand $\mathcal{O}_{L}(-2)[20,2.3]$; thus 
$\psi(f)=q \in H^{0} \mathcal{O}_{L}(b+2)$ and in particular $q \neq 0$ since $W \not \subset Q$. Now $W \subset 2 Q$ and its associated triple has the form $\left\{Z_{W}, L, D\right\}$ for a zero-dimensional subscheme $Z_{W} \subset L$. Interpreting diagram (3) for $W$, the middle column is simply the top row of diagram (5), $\mathcal{L} \cong \mathcal{O}_{L}(b)$, and the map $\sigma$ is multiplication by $q$; in particular, $\operatorname{deg} Z_{W}=b+2$.

The triple for $C$ has form $\{Z, D, D\}(Z \subset D$ a zero-dimensional subscheme) and the inclusion $W \subset C$ induces a morphism from diagram (3) for $C$ to diagram (3) for $W$ in which the middle rows coincide. Thus the maps along the bottom row are surjective and when we assemble these along with their kernels we obtain the commutative diagram

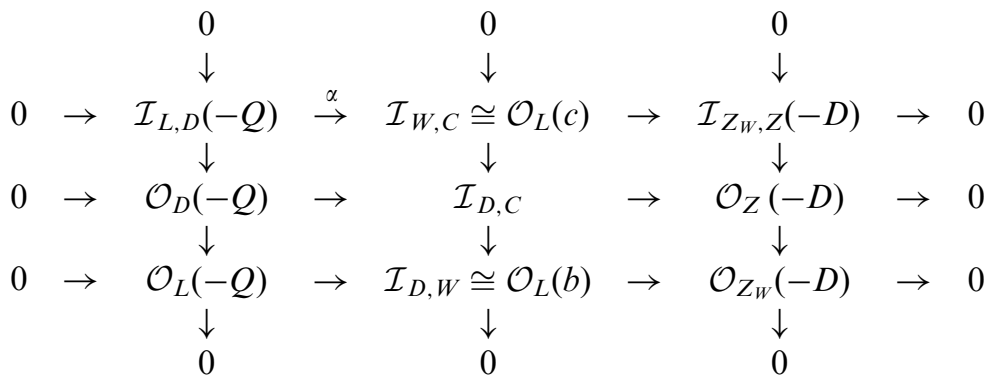

We immediately deduce the vanishing in statement 3 : indeed, the long exact cohomology sequence associated to the middle row shows that $H^{1}\left(\mathcal{I}_{D, C}\right)=0$, but in view of the discussion following diagram (3) and the fact that $D^{2}=0$ on $Q$ we have

$$
\mathcal{I}_{D, C} \cong \mathcal{O}_{D}(Z-Q) \cong \mathcal{O}_{D}(Z+D-Q)
$$

so that $H^{1}\left(\mathcal{O}_{D}(Z+D-Q)\right)=0$.

Now we analyze the map $\alpha$, which arises from the natural inclusion $\mathcal{I}_{L}(-2) \stackrel{f}{\hookrightarrow} \mathcal{I}_{L} \mathcal{I}_{D} \subset \mathcal{I}_{W}$ by taking quotients. Thus $\alpha$ factors as

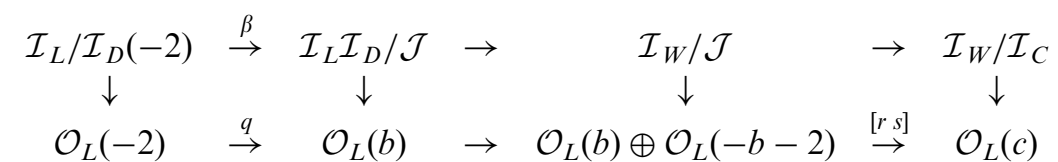

where $\mathcal{J}=\mathcal{I}_{Y} \mathcal{I}_{W}+\mathcal{I}_{D}^{2}$ and the vertical maps are isomorphisms via Lemma 2.2. We conclude that $\alpha$ is multiplication by the global section $q r \in H^{0}\left(L, \mathcal{O}_{L}(c+2)\right)$ so that $\mathcal{I}_{Z_{W}, Z} \cong \mathcal{O}_{L} /(q r)$. For a general 4 -line $C$ of type $(0, b, c)$, the form $q r$ has simple zeros.

Now twist diagram (6) by $\mathcal{O}_{D}(Q)=\mathcal{O}_{D}(-2)$ and apply $\mathfrak{H} \mathcal{O} \mathscr{M}_{\mathcal{O}_{D}}\left(-, \mathcal{O}_{D}\right)=(-)^{\vee}$. As $D$ is a local complete intersection (hence locally Gorenstein), $\mathcal{O}_{L}$ is a reflexive $\mathcal{O}_{D}$-module because it has local depth one $[9,1.6]$. In particular $\mathfrak{E} \mathscr{X} \mathscr{T}_{\mathcal{O}_{\mathrm{D}}}^{1}\left(\mathcal{O}_{\mathrm{L}}, \mathcal{O}_{\mathrm{D}}\right)=0$ and the columns remain exact. The leftmost column becomes

$$
0 \rightarrow\left(\mathcal{O}_{L}\right)^{\vee} \rightarrow\left(\mathcal{O}_{D}\right)^{\vee} \rightarrow\left(\mathcal{O}_{L}\right)^{\vee} \rightarrow 0
$$

(recall that $\left.\mathcal{I}_{L, D} \cong \mathcal{O}_{L}\right)$. It's easy to check on stalks that $\left(\mathcal{O}_{L}\right)^{\vee}$ is a locally free $\mathcal{O}_{L}$-module; the sequence above shows that the image of $1 \in H^{0}\left(\mathcal{O}_{D}\right)^{\vee}$ is a global 
generator, hence $\left(\mathcal{O}_{L}\right)^{\vee} \cong \mathcal{O}_{L}$. Since the dual of the reflexive sheaf $\mathcal{I}_{D, C}(Q) \cong \mathcal{O}_{D}(Z)$ is $\mathcal{I}_{Z, D}$, we obtain

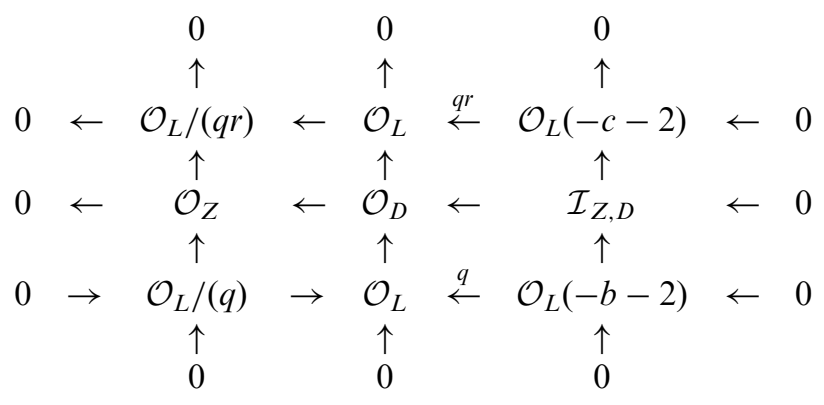

This diagram shows that $Z$ consists of $b+2$ double points supported at the isolated zeros of $q$ and $c-b$ reduced points.

We end this section showing that a general quasiprimitive 4-line as in Proposition 3.1 is a specialization on $X=2 Q$ of a disjoint union of double lines having arithmetic genera $-1-b$ and $-1-c$, a fact we will later use in Theorems 6.2 and 6.4. The idea is to lift families of triples to families of curves on $X$. In [24] we construct the Hilbert scheme $H_{z, r, p}$ of curves $C \subset X$ having triples $\{Z, R, P\}$ such that the Hilbert polynomial of $Z$ (resp. $R$ and $P$ ) is $z$ (resp. $r$ and $p$ ). If $D_{z, r, p}$ is the Hilbert flag scheme for the corresponding triples, there is a natural map $\pi: H_{z, r, p} \rightarrow D_{z, r, p}$ assigning a curve to its triple. The structure of this map is partially described as follows:

THEOREM 3.2 ([24], Theorem 3.1). Let $V \subset D_{z, r, p}$ be the open subset of triples $\{Z, R, P\}$ for which $H^{1} \mathcal{O}_{R}(Z+P-F)=0$. Then the induced map $\pi^{-1}(V) \rightarrow V$ is surjective and has the structure of an open immersion followed by an affine bundle projection.

We apply this machinery to prove:

PROPOSITION 3.3. Let $C$ be a general quasiprimitive 4-line of type $(0, b, c)$ lying on the double quadric surface $2 Q$. Then there is an irreducible flat family of curves on $2 Q$ with special fibre $C$ and general member a disjoint union of two double lines of respective arithmetic gener $a-1-b$ and $-1-c$.

Proof. As in Proposition 3.1, let $\{Z, D, D\}$ be the triple associated to $C$. Let $p_{1}, p_{2}, \ldots, p_{c-b}$ be the reduced points of $Z$, and choose planes $H_{j}$ transverse to $L$, one containing each double point $z_{j}$ of $Z$. Let $\left\{L_{t}: t \in \mathbb{P}^{1}\right\}$ be the family of rulings on $Q$ with $L_{0}=L$ and define

$$
Z_{t}=\left\{p_{1}, \ldots, p_{c-b}\right\} \cup \bigcup_{j=1}^{b+2}\left[H_{j} \cap\left(L_{t} \cup L_{0}\right)\right] .
$$


Setting $R_{t}=L_{t} \cup L_{0}$, we obtain a family of triples $\left\{Z_{t}, R_{t}, R_{t}\right\}$ specializing to $\{Z, D, D\}$. Moreover, for $t \neq 0$ we have the vanishing

$$
H^{1} \mathcal{O}_{R_{t}}\left(Z_{t}+D_{t}-Q\right) \cong H^{1}\left(\mathcal{O}_{L_{0}}(c+2-2)\right) \oplus H^{1}\left(\mathcal{O}_{L_{t}}(b+2-2)\right)=0 .
$$

Thus we obtain a map $\phi: \mathbb{P}^{1} \rightarrow D_{z, r, p}$ with image contained in the open set $V$ of triples for which $H^{1} \mathcal{O}_{R}(Z+P-Q)=0$. Over $V$ the natural map $\pi: H_{z, r, p} \rightarrow D_{z, r, p}$ is surjective and has the structure of an open immersion followed by an affine bundle projection by Theorem 3.2, hence the restriction $U=\pi^{-1}\left(\phi\left(\mathrm{P}^{1}\right)\right) \stackrel{\pi}{\rightarrow} \mathrm{P}^{1}$ has the same structure and is irreducible. We obtain the desired family $W$ by pulling back the universal family over $H_{z, r, p}$ to $U: C \in U$ by construction, and $W_{u}$ is a disjoint union of double lines of respective genera $-1-b$ and $-1-c$ whenever $\pi(u) \neq 0$ by Equation (4) (cf. [24, Example 2.8 (3)]).

\section{Curves of Degree Four with Large Speciality}

In this section we study curves which have large speciality. We express the speciality of a curve $C$ by its spectrum [28], which can be defined as the nonnegative function

$$
h_{C}(n)=\Delta^{2} h^{0}\left(\mathcal{O}_{C}(n)\right)=h^{0}\left(\mathcal{O}_{C}(n)\right)-2 h^{0}\left(\mathcal{O}_{C}(n-1)\right)+h^{0}\left(\mathcal{O}_{C}(n-2)\right) .
$$

We represent this by the t-uple of integers with exponents $\left\{n^{h_{C}(n)}\right\}$. The extremal curves, which by definition achieve the upper bounds on the Rao function $h^{1}\left(\mathcal{I}_{C}(n)\right)$ given in [17], also have the largest speciality and comprise an irreducible component $E \subset H_{4, g}$ of dimension $15-2 g$ [18, Theorems 2.5 and 3.7]. For $d=4$ and $g \leqslant 0$ they may be characterized as (a) nonplanar curves containing a plane cubic curve or (b) curves with spectrum $\{g\} \cup\{0,1,2\}$ [21,2.2].

Similarly, there are sharp upper bounds on the Rao function for nonextremal curves: the curves achieving these bounds are called subextremal and have spectrum $\left\{g+1,0,1^{2}\right\}$, although they are not characterized by this fact $[21,2.11,2.14$ and $2.15]$. We characterize curves of degree four with subextremal speciality as follows:

LEMMA 4.1. Let $C$ be a curve of degree 4 and genus $g \leqslant-2$. Then $C$ has spectrum $\left\{g+1,0,1^{2}\right\}$ if and only if $C$ contains a subcurve $T$ of degree 3 and genus 0.

Proof. If $C$ contains such a curve $T \in H_{3,0}$, then the principal spectrum spectrum $\left\{0,1^{2}\right\}$ of $T$ is contained in that of $C[28, \S 3]$ and the remaining element $g+1$ is determined by the genus of $C$.

On the other hand, suppose that $C$ has spectrum $\left\{g+1,0,1^{2}\right\}$. Then $h^{0} \mathcal{O}_{C}$ $(g+1)=1$ and choosing $0 \neq \alpha \in H^{0} \mathcal{O}_{C}(g+1)$ gives a map $\mathcal{O}_{C} \rightarrow \mathcal{O}_{C}(g+1)$ with image $\mathcal{O}_{D}$ for some closed subscheme $D \subset C$. The local depth of $D$ is one because $\mathcal{O}_{D} \subset \mathcal{O}_{C}(g+1)$, hence $D$ is a locally Cohen-Macaulay curve. The inclusion above also shows that $h^{0} \mathcal{O}_{D}(1) \leqslant 2$ : for $g<-2$ this is because $h^{0} \mathcal{O}_{C}(g+2)=2$. If $g=-2$, then $h^{0} \mathcal{O}_{C}=3$ and the inclusion $\mathrm{H}^{0}\left(\mathcal{O}_{D}(1)\right) \subset \mathrm{H}^{0}\left(\mathcal{O}_{C}\right)$ is strict, as otherwise we obtain 
a surjection $\mathcal{O}_{D}(1) \rightarrow \mathcal{O}_{C}$, which is absurd. It follows that $D=L$ is a line. This yields an exact sequence

$$
0 \rightarrow \mathcal{O}_{L}(-g-1) \rightarrow \mathcal{O}_{C} \rightarrow \mathcal{O}_{T} \rightarrow 0
$$

for a closed subscheme $T \subset C$ of degree 3 and genus 0 .

If $T$ is not purely one-dimensional, then the purely one-dimensional part $P \subset T$ is planar because $g(P)>0$ and $\operatorname{deg} P=3$ [10,3.1], but this is not possible because $C$ is not extremal. Thus $T$ is locally Cohen-Macaulay, finishing the proof.

PROPOSITION 4.2. Let $C$ be a curve of degree 4 and genus $g \leqslant-1$ having spectrum $\left\{g+1,0,1^{2}\right\}$. Then there is a line $L$ such that the Rao module $M_{C}=H_{*}^{1}\left(\mathcal{I}_{C}\right)$ is a graded module over the coordinate ring $S=H_{*}^{0}\left(\mathcal{O}_{L}\right)$ with resolution

$$
0 \rightarrow S(-j) \oplus S(j-5+g) \stackrel{\sigma}{\rightarrow} S(-2)^{\oplus 3} \rightarrow S(-g-1) \rightarrow M_{C} \rightarrow 0
$$

for some integer $2 \leqslant j \leqslant n(g)=\left\lfloor\frac{5-g}{2}\right\rfloor$. The cohomology of $C$ is determined by the integer $j$ and we denote the corresponding family of curves by $H_{j}$.

Proof. First we suppose that $g \leqslant-2$; then $C$ contains a curve $T$ of degree 3 and genus 0 by Lemma 4.1. In particular, $T$ is arithmetically Cohen-Macaulay and the total ideal $I_{T}$ is generated by three quadrics [10,3.5]. Moreover, the proof of Lemma 4.1 produces a line $L$ such that $\mathcal{I}_{T, C} \cong \mathcal{O}_{L}(-g-1)$. Thus we obtain the composite surjection $\mathcal{O}(-2)^{\oplus 3} \rightarrow \mathcal{I}_{T} \rightarrow \mathcal{O}_{L}(-g-1)$ : factoring this map through $\mathcal{O}_{L}(-2)^{\oplus 3}$ and writing the kernel of the induced map as $\mathcal{O}_{L}(-j) \oplus \mathcal{O}_{L}(j-5+g)$ for some integer $j \in[2,\lfloor(5-g) / 2\rfloor]$, we obtain resolution (10).

Now assume that $g=-1$. The spectrum shows that $C$ is neither ACM (because $\left.h_{C}(0)>1\right)$ nor extremal, hence [21, Theorem 2.11] gives the bounds $h^{1} \mathcal{I}_{C} \leqslant 1$, $h^{1} \mathcal{I}_{C}(1) \leqslant 2$ and $h^{1} \mathcal{I}_{C}(2) \leqslant 1$. The first two of these are equalities in view of the Euler characteristics (since $h^{3} \mathcal{I}_{C}=h^{3} \mathcal{I}_{C}(1)=0$ ) and the vanishing $h^{2} \mathcal{I}_{C}(1)=0$, which may be read from the spectrum. If $h^{1} \mathcal{I}_{C}(2)=1$, then $C$ is subextremal by definition and has (a degenerate form of) resolution (10) with $j=2$.

If $h^{1} \mathcal{I}_{C}(2)=0$, then $\mathcal{I}_{C}$ is 3 -regular (hence $h^{1} \mathcal{I}_{C}(n)=0$ for $n \geqslant 2$ ) and $h^{0} \mathcal{I}_{C}(2)=h^{2} \mathcal{I}_{C}(2)=0$. The condition $h^{1} \mathcal{I}_{C}=1$ implies that $h^{1} \mathcal{I}_{C}(n)=0$ for $n<0$ $[19,2.8]$, hence the Rao module $M_{C}$ has a generator $m$ in degree 0 . If $m$ does not generate $M_{C}$ as a module over the homogeneous coordinate ring $S=k[x, y, z, w]$ of $\mathrm{P}^{3}$, then $m$ is annihilated by 3 independent linear forms, which implies that $C$ lies on a quadric by [27, 3.4.5], a contradiction. Thus $m$ generates the Rao module and $M_{C} \cong S /\left(x, y, z^{2}, z w, w^{2}\right)$ after a change of coordinates, so that $M_{C}$ has resolution (10) for $I_{L}=(x, y)$ with $j=3$.

Finally, we describe the families $H_{j}$ and how they fit together in the Hilbert scheme. We let $G_{4} \subset H_{4, g}$ denote the closed family of thick 4-lines. By Proposition $2.1 G_{4}$ is irreducible of dimension $9-3 g$. 
PROPOSITION 4.3. For fixed $g \leqslant-1$, let $H_{j}$ be the family of curves defined in Proposition 4.2. Then

(1) The family $\mathrm{H}_{2}$ is irreducible and meets $G_{4}$. It consists of subextremal curves and has dimension $13-2 g$ (resp. 16 if $g=-1$ ).

(2) The family $\mathrm{H}_{3}$ is irreducible and meets $G_{4}$. It has dimension $13-2 g$ (resp. 16 if $g=-1)$. If $g=-1$ or -2 , then $G_{4} \subset \overline{H_{3}}$.

(3) Suppose $g \leqslant-3$. The families $H_{j}$ are contained in $G_{4}$ for $3<j \leqslant n(g)$ and $G_{4}=\overline{H_{n(g)}}$.

Proof. We consider the last statement first: suppose that $C \in H_{j}$ for $3<j \leqslant n(g)$. Sequence (10) shows that $h^{1} \mathcal{I}_{C}(3)=-g-3$, hence $h^{0} \mathcal{I}_{C}(3)=4$. If $L$ and $T$ are as in the proof of Proposition 4.1 above, then $\operatorname{dim}\left(I_{L} I_{T}\right)_{3} \leqslant 4$ because $I_{L} I_{T} \subset I_{C}$ and so $I_{T}=I_{L}^{2}$ by Lemma 4.5 below. It follows that $C$ is a thick 4-line supported on $L$, hence $H_{j} \subset G_{4}$. As we saw in Proposition 2.1, the thick 4-lines supported on $L$ are parametrized by the open subset

$$
U \subset \operatorname{Hom}_{\mathcal{O}}\left(\mathcal{I}_{T}, \mathcal{O}_{L}(-g-1)\right) \cong \operatorname{Hom}_{\mathcal{O}_{L}}\left(\mathcal{O}_{L}(-2)^{3}, \mathcal{O}_{L}(-g-1)\right)
$$

corresponding to surjective maps. For $j=2$ and 3, the specific surjections given by $\left(w^{1-g}, w^{3-g-j} z^{j-2}, z^{1-g}\right)$ show that $G_{4}$ meets $H_{j}$. Since $I_{C}$ is determined by its image in $I_{T} / I_{L} I_{T} \cong S_{L}(-2)^{3}$ (and hence by the image of the first map in sequence (10) above), we find by counting dimensions that $H_{j}$ is irreducible of dimension $5+2 j-2 g$ (except if $2 j=5-g$, when the dimension is $4+2 j-2 g$ ). For $j=n(g)=\left\lfloor\frac{5-g}{2}\right\rfloor$, the closure of $H_{n(g)}$ is irreducible of dimension $9-3 g$, hence is equal to $G_{4}$.

If $j=2$, then $H_{j}$ consists of subextremal curves: indeed, for $C \in H_{2}$, resolution (10) gives Rao function $\rho_{C}(n)=\left(\begin{array}{c}n-g \\ 1\end{array}\right)-2\left(\begin{array}{c}n-1 \\ 1\end{array}\right)+\left(\begin{array}{c}n+g-2 \\ 1\end{array}\right)$, which agrees with the subextremal bound in [21, 2.11]. In particular, these curves are obtained from extremal curves of degree 2 and genus $g^{\prime}=g-1$ by a height one biliaison on a quadric surface $[21,2.14]$. Let then $\gamma, \rho$ (resp. $\gamma^{\prime}, \rho^{\prime}$ ) be the gamma and Rao functions for the extremal curves of degree 2 and genus $g-1$ (resp. subextremal curves of degree 4 and genus $g$ ). Letting $\mathcal{B}_{\gamma, \rho, 2,1}$ denote the universal biliaison scheme of Martin-Deschamps and Perrin [16, VII § 4], we have smooth irreducible projections

$$
\begin{aligned}
& \mathcal{B}_{\gamma, \rho, 2,1} \stackrel{q_{2}}{\rightarrow} H_{\gamma^{\prime}, \rho^{\prime}} \\
& \quad q_{1} \downarrow \\
& E=H_{\gamma, \rho}
\end{aligned}
$$

to the spaces $H_{\gamma, \rho}$ (resp. $H_{\gamma^{\prime}, \rho^{\prime}}$ ) of curves with constant cohomology. The family $E=H_{\gamma^{\prime}, \rho^{\prime}}$ of extremal curves is irreducible of dimension $7-2 g[18,2.5]$ and using [16, VII, 4.8] we compute that the fibre dimension of $q_{1}$ is 8 (resp. 9 if $g=-1$ ) and the fibre dimension of $q_{2}$ is 2 , hence the family $H_{\gamma, \rho}$ of subextremal curves is irreducible of dimension $13-2 g$ (resp. 16 if $g=-1$ ). 
For $j=3$, we take an indirect approach. Consider the family of arithmetically Cohen-Macaulay curves $D$ with resolution of the form

$$
0 \rightarrow \mathcal{O}(2 g-1) \oplus \mathcal{O}(g-2) \rightarrow \mathcal{O}(g-1)^{3} \rightarrow \mathcal{I}_{D} \rightarrow 0
$$

It is irreducible of dimension $2\left(\begin{array}{c}-g+3 \\ 3\end{array}\right)+(\underset{2}{-g+2})+2$ (resp. 12 if $g=-1$ ) by [6] and the general member is smooth and irreducible (the numerical character has no gaps).

Let $D$ be a general such curve in this family. Then a general (disjoint) union $D \cup L$ with a line $L$ lies on an integral surface of degree $-g+2$; To see this, we consider the map

$$
\tau: \mathbb{P}^{3}-D-L \stackrel{\Delta}{\rightarrow} \mathbb{P}^{3}-D \times \mathbb{P}^{3}-L \rightarrow \mathbb{P}^{2} \times \mathbb{P}^{1}
$$

given by the linear systems $\mathbb{P} H^{0} \mathcal{I}_{D}(-g+1)$ and $\mathbb{P} H^{0} \mathcal{I}_{L}(1)$. The fibres of $\tau$ are the intersections $\left\{S \cap H: S \in H^{0} \mathcal{I}_{D}(-g+1), H \in H^{0} \mathcal{I}_{L}(1)\right\}$, which are generally of dimension one, and hence the image of $\tau$ has dimension two. Composing with the Segre embedding $\mathbb{P}^{2} \times \mathbb{P}^{1} \hookrightarrow \mathbb{P}^{5}$, we apply Jouanolou's Bertini theorem $[15,6.10]$ to see that the general surface of degree $-g+2$ containing $D \cup L$ is irreducible. Furthermore, the resolution for $\mathcal{I}_{D}$ shows that $H^{1} \mathcal{O}_{D \cup L}((-g+2)-4-(g+1)) \neq 0$ and we find that [16, III, 2.7(b)] $D \cup L$ can be bilinked on a surface of degree $-g+2$ with height $g+1$ to a curve $C$, which lies in $H_{3}$ by direct calculation.

Let $\gamma, \rho$ (resp. $\gamma^{\prime}, \rho^{\prime}$ ) be the gamma and Rao functions for curves in $H_{3}$ (resp. $D \cup L$ ). Letting $\mathcal{B}_{\gamma, \rho,-g+2,-g-1}$ be the universal biliaison scheme [16, VII Section 4], we obtain smooth irreducible projections

$$
\begin{gathered}
\mathcal{B}_{\gamma, \rho,-g+2,-g-1} \stackrel{q_{2}}{\rightarrow} H_{\gamma^{\prime}, \rho^{\prime}} \\
q_{1} \downarrow \\
H_{3}=H_{\gamma, \rho}
\end{gathered}
$$

From the last paragraph, the image of $q_{2}$ is dense in the irreducible component consisting of the closure of the family of disjoint unions $D \cup L$ considered above. Using the resolutions given, we compute the dimension of the fibres of $q_{1}$ and $q_{2}$ via [16, VII 4.8] and conclude that $H_{3}$ is irreducible of dimension $13-2 g$ (resp. 16 if $g=-1)$.

Remark 4.4. One can check by a dimension count that the general members of the families $H_{2}$ and $H_{3}$ are described as follows.

1. For $g=-1$, the general member of $H_{2}$ is a disjoint union of conics. For $g \leqslant-2$, the general member of $H_{3}$ is the union of a double line $Z$ of genus $g-2$ and two disjoint lines $L_{1}$ and $L_{2}$, each meeting $Z$ in a scheme of length 2 .

2. For $g=-1$, the general member of $H_{3}$ is a disjoint union of a line and a twisted cubic curve. For $g \leqslant-2$, the general member of $H_{3}$ is the union of a double line of genus $g-1$ and a smooth conic meeting in a scheme of length 2 .

The following lemma and its proof are well known: 
LEMMA 4.5. Let $L \subset \mathbb{P}^{N}$ be a linear subvariety of codimension two and let $V \subset H^{0}\left(\mathbb{P}^{N}, \mathcal{O}(d)\right)$ be a subspace of dimension $r$ generating the homogeneous ideal I. Then the image $W$ under the multiplication map $V \otimes H^{0}\left(\mathbb{P}^{N}, \mathcal{I}_{L}(1)\right) \rightarrow$ $H^{0}\left(\mathbb{P}^{N}, \mathcal{O}(d+1)\right)$ satsifies $\operatorname{dim}(W) \geqslant r+1$ with equality if and only $I=I_{L}^{r-1} f$ for some form $f$ of degree $d-r+1$.

Proof. Let $S=\operatorname{Sym} H^{0}\left(\mathrm{P}^{N}, \mathcal{I}_{L}(1)\right) \cong k[x, y]$ be the symmetric algebra and set $\mathbb{P}^{1}=\operatorname{Proj}(S)$. Let $\mathcal{F}$ denote the image of the sheafification of the natural map $V \otimes_{k} S \rightarrow \bigoplus_{n} \mathrm{H}^{0}\left(\mathbb{P}^{N}, \mathcal{O}(n)\right)$ of free graded $S$-modules. This yields an exact sequence $0 \rightarrow \mathcal{E} \rightarrow \mathcal{O}_{\mathrm{P}^{1}}^{r} \rightarrow \mathcal{F} \rightarrow 0$ of locally free $\mathcal{O}_{\mathrm{p}^{1}}$-modules. Since $h^{0}(\mathcal{E})=0$ by hypothesis and we have $\operatorname{rank}(\mathcal{E}) \leqslant r-1$, so

$$
\mathcal{E} \cong \bigoplus_{i=1}^{s} \mathcal{O}_{\mathrm{P}^{1}}\left(-a_{i}\right)
$$

with $s \leqslant r-1$ and $a_{i}>0$, hence $\mathrm{h}^{0}(\mathcal{E}(1)) \leqslant r-1$. Since $\mathrm{H}^{0}(\mathcal{E}(1))$ is the kernel of the surjection $V \otimes S_{1} \rightarrow W$, we see that $\operatorname{dim}(W) \geqslant r+1$ with equality if and only if $s=r-1$ and $a_{i}=1$ for $1 \leqslant i \leqslant r-1$, which is equivalent to saying that $\mathcal{F} \cong \mathcal{O}_{\mathrm{P}^{1}}(r-1)$.

\section{Triple Lines Union a Line}

In this section we are interested in unions $C=W \cup L$ of a quasi-primitive triple line $W$ of type $(a, b)$ and a reduced line $L$, where $W \cap L$ is nonempty. The arithmetic genus of $C$ is given by

$$
g(C)=-3 a-3-b+\text { length }(W \cap L) .
$$

Fix $g$ and $a \geqslant 0$ and let $Z \subset W$ denote the underlying double line of genus $-1-a$. Then we have four families of such curves $C$ in $H_{4, g}$ as shown in Table I.

The main results of this section are that families $F_{1}$ and $F_{3}$ are irreducible (Proposition 5.2) and that the other two families lie in their closures (Proposition 5.4).

Our arguments hinge on the following construction: fix a double line $Z \subset \mathrm{P}^{3}$ of type $a \geqslant 0$ with support $Y$ and let $b \geqslant 0$ be an integer. Let $h \in H^{0} \mathcal{I}_{Z}(a+b+2)$ be the equation of a surface $S$ which does not contain the first infinitesimal neighborhood $Y^{(2)}$ of $Y$. Removing the embedded points from the scheme cut out by

Table I. Families of unions $W \cup L, W$ a triple line of type $(a, b)$

\begin{tabular}{llll}
\hline Label & Length $(W \cap L)$ & Length $(Z \cap L)$ & Relation \\
\hline$F_{1}$ & 3 & 2 & $b=-3 a-g$ \\
$F_{2}$ & 2 & 2 & $b=-3 a-g-1$ \\
$F_{3}$ & 2 & 1 & $b=-3 a-g-1$ \\
$F_{4}$ & 1 & 1 & $b=-3 a-g-2$
\end{tabular}


$I=\left(I_{Y} I_{Z}, h\right)$ yields a locally Cohen-Macaulay curve $W$ supported on $Y$. Since $Y^{(2)} \not \subset S$, the surface $S$ is smooth at a general point $p$ of $Y$, so as in Section 2 we may find local equations $s=h_{p}$ and $t$ for $Y$ at $p$ such that $\mathcal{I}_{D, p}=\left(s, t^{2}\right)$, and $\mathcal{I}_{W, p}=\left(s, t^{3}\right)$. It follows that $W$ is quasiprimitive of degree three. Thus we obtain the map

$$
\Phi:\left\{h \in H^{0}\left(\mathcal{I}_{Z}(a+b+2)\right): h \notin I_{Y}^{2}\right\} \rightarrow\{\text { Quasiprimitive 3-lines } W \supset Z\} .
$$

LEMMA 5.1. Let $\Phi$ be the map above. Then

(1) The image of $\Phi$ is the set of quasiprimitive 3-lines $W \supset Z$ of type $\left(a, b^{\prime}\right)$ with $b^{\prime} \leqslant b$.

(2) For $h$ as above, set $I=\left(I_{Y} I_{Z}, h\right)$. Then the following are equivalent.

(a) $W=\Phi(h)$ has type $(a, b)$.

(b) The scheme defined by I is locally Cohen-Macaulay.

(c) $h$ is irreducible modulo $I_{Y} I_{Z}$.

If any of these conditions hold, then $I=I_{W}$ is saturated.

Proof. For $h \in\left\{\left(I_{Z}\right)_{a+b+2}: h \notin I_{Y}^{2}\right\}$, let $W$ be the purely one-dimensional part of the scheme $V$ defined by the ideal $I=\left(I_{Y} I_{Z}, h\right)$. Since $W$ is quasi-primitive and contains $Z, W$ has type $\left(a, b^{\prime}\right)$ for some $b^{\prime} \geqslant a$ and the total ideal may be written $I_{W}=\left(I_{Y} I_{Z}, h^{\prime}\right)$ with $h^{\prime}$ of degree $a+b^{\prime}+2$ by [20, 2.3]. The inclusions $\left(I_{Y} I_{Z}, h\right) \subset I_{V} \subset I_{W}$ now show that $b^{\prime} \leqslant b$. On the other hand, if $W$ is a quasiprimitive 3-line of type $\left(a, b^{\prime}\right)$ with $b^{\prime} \leqslant b$, then writing $I_{W}=\left(I_{Y} I_{Z}, h^{\prime}\right)$ as above and choosing a hypersurface $F$ of degree $b-b^{\prime}$ with equation $f$ meeting $Z$ properly, we see that $\Phi\left(f h^{\prime}\right)=W$.

For the equivalences in statement 2 , let $C$ be the scheme defined by $I=\left(I_{Y} I_{Z}, h\right)$ so that $W=\Phi(h)$ is obtained from $C$ by removing possible embedded points.

$(a) \Rightarrow(b)$ If $W$ has type $(a, b)$, then by $[20,2.3]$ the total ideal for $W$ takes the form $I_{W}=\left(I_{Y} I_{Z}, h^{\prime}\right)$ with $\operatorname{deg} h^{\prime}=a+b+2=\operatorname{deg} h$. The inclusions $I \subset I_{C} \subset I_{W}$ show that all three ideals are equal, so $C=W$ is locally Cohen-Macaulay.

(b) $\Rightarrow$ (c) Suppose that $h=h^{\prime} t$ modulo $I_{Y} I_{Z}$. If $T$ is the surface with equation $t$, then we may assume $T$ meets $Y$ properly (since if both $t \in I_{Y}$ and $h^{\prime} \in I_{Y}$, then $h \in I_{Y}^{2}$, contrary to hypothesis). In this case $\left(I_{Y} I_{Z}, t\right)$ defines a scheme of length $4 \operatorname{deg} T$ (because $I_{Y} I_{Z}$ defines a locally Cohen-Macaulay 4-line) and if $C$ is locally CohenMacaulay, then $(I, t)$ defines a scheme of length $3 \operatorname{deg} T$; since $\left(I_{Y} I_{Z}, t\right)=(I, t)$, we must conclude that $C$ is not locally Cohen-Macaulay.

$(c) \Rightarrow(a)$ If $W$ has type $\left(a, b^{\prime}\right)$ for $b^{\prime}<b$, then $I_{W}=\left(I_{Y} I_{Z}, h^{\prime}\right)$ with $\operatorname{deg} h^{\prime}=a+b^{\prime}+2$. The inclusion $I \subset I_{W}$ shows that there exists $t$ of degree $b-b^{\prime}$ such that $h=h^{\prime} t$ modulo $I_{Y} I_{Z}$.

PROPOSITION 5.2. With the notation of Table I, we have

(1) The family $F_{1} \subset H_{4, g}$ is irreducible of dimension $11-2 g-a$ if $0 \leqslant a \leqslant-\frac{g}{3}$ and empty if $a>-(g / 3)$. 
(2) The family $F_{3} \subset H_{4, g}$ is irreducible of dimension $10-2 g-a$ if $0 \leqslant a<(-g-1) / 3$ and empty if $a \geqslant(-g-1) / 3$.

Proof. We first prove statement 1, then indicate the changes to obtain statement 2. Let $H \subset H_{3,-a}$ be the family of unions $Z \cup_{2 P} L$. By [20, 3.2(a)], $H$ is irreducible of dimension $9+2 a$. For $b=-3 a-g$ we interpret $H^{0} \mathcal{O}_{\mathrm{p}^{3}}(a+b+2)$ as an affine scheme. Pulling back the universal family over $H$ we obtain a diagram

$$
\begin{gathered}
\mathcal{Z} \cup \mathcal{L} \subset \mathbb{P}^{3} \times H \times H^{0}\left(\mathcal{O}_{\mathbb{P}^{3}}(a+b+2)\right) \\
\searrow \\
H \times H^{0}\left(\mathcal{O}_{\mathbb{P}^{3}}(a+b+2)\right) .
\end{gathered}
$$

Consider the closed subset

$$
V=\left\{(Z \cup L, h) \in H \times H^{0}\left(\mathcal{O}_{\mathrm{P}}^{3}(a+b+2)\right): h \in I_{Z} \cap\left(I_{Y}^{3}, I_{L}\right)\right\}
$$

with first projection $V \stackrel{p_{1}}{\rightarrow} H$. The fibres of $p_{1}$ are vector subspaces of dimension $\left(\begin{array}{c}a+b+5 \\ 3\end{array}\right)-3 a-2 b-7$. Indeed, after a change of coordinates we may write $I_{Z}=\left(x^{2}, x y, y^{2}, x g-y f\right)([20,1.4(\mathrm{c})])$ and $I_{L}=(x, z)$, when the fibre is identified with the kernel $K$ of the composite map

$$
H^{0}\left(\mathcal{I}_{Z}(a+b+2)\right) \hookrightarrow\left(x, z, y^{2}\right)_{a+b+2} \rightarrow\left(\left(x, z, y^{2}\right) /\left(x, z, y^{3}\right)\right)_{a+b+2}
$$

(the inclusion has the correct target because $Z$ meets $L$ in the double point $2 P$ ). Since $\mathcal{I}_{Z}$ is $(a+2)$-regular,

$$
h^{0}\left(\mathcal{I}_{Z}(a+b+2)\right)=\chi \mathcal{I}_{Z}(a+b+2)=\left(\begin{array}{c}
a+b+5 \\
3
\end{array}\right)-3 a-2 b-6
$$

and $p_{1}: V \rightarrow H$ is an affine bundle with fibres of dimension $\left({ }^{a+b+5}\right)-3 a-2 b-7$. In particular, $V$ is irreducible.

Consider the open subset $U=\left\{(Z \cup L, h) \in V: h \notin I_{Y}^{2}\right\}$. The correspondence of Lemma 5.1 shows that elements of $U$ determine unions $W \cup L$ in which $W$ is a quasiprimitive 3-line. If $\mathcal{S} \subset \mathbb{P}^{3} \times U$ is the family of surfaces with equation $h$, we obtain flat families

$$
\begin{aligned}
\mathcal{Y}, \mathcal{Z} \cup \mathcal{L}, \mathcal{S} \subset \mathbb{P}^{3} & \times U \\
\searrow & \downarrow \\
& U
\end{aligned}
$$

where $\mathcal{Y}$ is the support of $\mathcal{Z}$. The ideal sheaf $\mathcal{I}_{F}=\mathcal{I}_{\mathcal{Y}} \mathcal{I}_{\mathcal{Z}}$ defines a flat subscheme $F \subset \mathbb{P}^{3} \times U$ whose fibres are thick 4-lines of genus $-2 a-1$ (easy calculation on the fibres). The subscheme $\mathcal{W} \subset \mathbb{P}^{3} \times U$ defined by the ideal sheaf $\mathcal{I}_{\mathcal{W}}=\mathcal{I}_{\mathcal{Y}} \mathcal{I}_{\mathcal{Z}}+\mathcal{I}_{\mathcal{S}}$ is also flat over $U$. To see this, observe that the relative ideal sheaf $\mathcal{I}_{\mathcal{W}, F}=\mathcal{I}_{\mathcal{W}} / \mathcal{I}_{F}$ is an $\mathcal{O}_{y}$-module, whose fibre over the point $u=(Z \cup L, h)$ is generated by the equation $h$. This gives a right exact sequence

$$
\mathcal{O}_{\mathcal{Y}_{u}}(-a-b-2) \stackrel{h}{\rightarrow} \mathcal{O}_{F_{u}} \rightarrow \mathcal{O}_{\mathcal{W}_{u}} \rightarrow 0 .
$$

From the choice of $V$ and $U$ (and the construction of the map $\Psi$ ) we know that the fibre $\mathcal{W}_{u}$ is a scheme of degree 3. In considering the degree of the Hilbert 
polynomials, we see that the map $h$ is injective and that $\mathcal{W}_{u}$ has Hilbert polynomial $p(z)=3 z+3 a+b+3$ independent of $u$, so $\mathcal{W}$ is flat [8, III, 9.9].

Finally, let $U^{\prime} \subset U$ be the open set for which the fibres of $\mathcal{W}$ are locally CohenMacaulay, taken with the induced reduced scheme structure. This is precisely the set for which the fibres of $\mathcal{W}$ are quasiprimitive 3-lines of type $(a, b)$ by Lemma 5.1. The curves used in the proof of Corollary 5.5 show that $U^{\prime}$ is non-empty; the double line $Z$ with total ideal $I_{Z}=\left(x^{2}, x y, y^{2}, x z^{a+1}-y w^{a+1}\right)$ has the line $L=\{x=w=0\}$ as a double tangent and $h=z^{b}\left(x z^{a+1}-y w^{a+1}\right)-x^{2} w^{a+b}=0$ satisfies the conditions above. The definition of $V$ above makes it clear that the fibres of $\mathcal{W}$ meet the lines $L$ in triple points, so the family $\mathcal{W} \cup \mathcal{L}$ is also flat over $U^{\prime}$. The universal property of the Hilbert scheme gives a map $U^{\prime} \rightarrow H_{4,-3 a-b}$ whose image is precisely the family $F_{1}$ of unions $W \cup_{3 P} L$. In particular, $F_{1}$ is irreducible.

The structure of the map $U^{\prime} \rightarrow H$ shows that $U^{\prime}$ has dimension $\left(\begin{array}{c}a+b+5 \\ 3\end{array}\right)-a-2 b+2$. On the other hand, if $W$ is a triple line arising in the construction above, then $\mathcal{I}_{W}$ is $(a+b+2)$-regular [20, 2.4], hence $\operatorname{dim} H^{0}\left(\mathcal{I}_{W}(a+b+2)\right)=$ $\left({ }_{3}^{a+b+5}\right)-6 a-4 b-9$. Subtracting this redundancy shows that the family has dimension $5 a+2 b+11=11-2 g-a$.

The proof of statement 2 goes through via the same outline. The main differences are as follows. The family $H \subset H_{3,-a-1}$ is now the family of unions $Z \cup_{P} L$, which is irreducible of dimension $10+2 a$ by [20, 3.2(b)]. In definition (11) of $V, I_{Y}^{3}$ is replaced by $I_{Y}^{2}$ and in map (12) the exponents of $y$ should be reduced by one. To see that $U^{\prime}$ is nonempty, we can use the same triple line $W$ as in the proof above, but instead use the line $L$ given by $\{x=z=0\}$. The remaining modifications are clear.

Remark 5.3. Since the family of triple lines $W$ is irreducible of dimension $10+5 a+2 b[20,2.6]$, we expect that the natural map $\left\{W \cup_{3 P} L\right\} \stackrel{F}{\rightarrow}\{W\}$ which forgets the line $L$ has generically one dimensional fibres. However, there are triple lines $W$ for which the fibre $F^{-1}(W)$ has larger dimension. For example, the triple lines constructed in characteristic $p>0$ by Hartshorne [10,2.3] have a two-dimensional family of triple tangent lines.

PROPOSITION 5.4. With the notation above, we have

(1) $F_{2} \subset \bar{F}_{1}$ if $0 \leqslant a<\frac{-g-1}{3}$ and is otherwise empty.

(2) $F_{4} \subset \bar{F}_{3}$ if $0 \leqslant a<\frac{-g-1}{3}$ and is otherwise empty.

Proof. Let $W_{0} \cup_{2 P} L$ be a curve in the family $F_{2}$, so that the underlying double line $Z \subset W_{0}$ satisfies length $(Z \cap L)=2$. If $W_{0}$ has type $(a,-3 a-g-1)$ and support $Y$; we may write $I_{Y}=(x, y), I_{Z}=\left((x, y)^{2}, x g-y f\right), I_{L}=(x, z)$ and $I_{W_{0}}=\left(I_{Y} I_{Z}, h_{0}\right)$ in suitable coordinates [20, 2.3]. If $K \subset H^{0}\left(\mathcal{I}_{Z}(-2 a-g+2)\right)$ is the vector subspace considered in the proof above, then $z h_{0} \in K$. Fixing a member $(Z \cup L, h) \in U^{\prime}$ as above, the deformation $(1-t) z h_{0}+t h$ gives a map $\mathrm{A}^{1} \stackrel{\psi}{\rightarrow} K$, which yields $\psi^{-1}\left(U^{\prime}\right) \rightarrow H_{4, g}$. This extends to a map $\bar{\psi}: T=\psi^{-1}\left(U^{\prime}\right) \cup\{0\} \rightarrow$ Hilb $_{4}^{\mathrm{g}}$ into the full 
Hilbert scheme: by construction, it's clear that the limit curve $\bar{\psi}(0)$ contains $W_{0} \cup L$. Since this curve has genus $g$, it is equal to $\bar{\psi}(0)$, completing the proof. The limit of the triple lines $W_{t}$ is the triple line $W_{0}$ along with an embedded point, which is conveniently covered up by the line $L$. Statement 2 is similar.

COROLLARY 5.5. The closure of family $F_{1, a}$ in $H_{4, g}$ contains extremal curves.

Proof. Following [20, 3.6], the family of ideals $I_{t}$ below give a deformation from a triple line $W$ of type $(a, b)$ to an extremal curve of the same arithmetic genus.

$$
I_{t}=\left((x, y)^{3},(x, y)\left(x z^{a+1}-t y w^{a+1}\right), z^{b} t^{2}\left(x z^{a+1}-t y w^{a+1}\right)-x^{2} w^{a+b}\right) .
$$

We simply observe that the line $L=\{x=w=0\}$ is triple tangent to the triple line $W_{t}$ defined by $I_{t}$ for all $t \neq 0$ and that this same line is a triple tangent to the limit extremal curve having ideal

$$
I_{0}=\left(x^{2}, x y, y^{3}, x z^{3 a+b+3}-y^{2} w^{3 a+b+2}\right) .
$$

Remark 5.6. The closure of the family $F_{3}$ above forms an irreducible component of the Hilbert scheme (Theorem 6.2) with one exception: We will show that the curves $C_{0}=W_{0} \cup_{2 P} L \in F_{3}$ with $W_{0}$ of type $(0, b)$ are flat limits of curves $C_{t}=L \cup_{2 P} Z_{t} \cup L_{t}$, where $Z_{t}$ is a double line and $L_{t}$ is a line disjoint from $L$ (compare with [20, 3.3]).

Recall that the underlying double line $Z_{0} \subset W_{0}$ lies on a smooth quadric with equation $q=0[20,1.5]$ and that the ideal of $W_{0}$ may be written $I_{W_{0}}=\left((x, y)^{3}\right.$, $\left.x q, y q, h=p q-a x^{2}-b x y-c y^{2}\right)[20,2.6]$. The double point $2 P=W_{0} \cap L$ is not contained in $Z_{0}$ since $C_{0} \in F_{3}$.

Letting $L_{t}$ be the ruling $\{x+w t=y+z t=0\}$ of $Q$ for $t \in \mathbb{A}^{1}$, the flat family $D_{t}=L_{t} \cup L_{0}$ has limit $D_{0}=Z_{0}$ (linear equivalence on $Q$ ) and the flat family of subschemes $E_{t}=D_{t} \cup 2 P$ has limit $Z_{0} \cup 2 P$. Since $\operatorname{dim} H^{0} \mathcal{I}_{E_{t}}(-g+1)$ is constant, $\pi_{*}\left(\mathcal{I}_{E}(-g+1)\right)$ is locally (and hence globally) free on $\mathrm{A}^{1}$ by Grauert's theorem [8, III, 12.9], where $E \subset \mathbb{P}^{3} \times \mathbb{A}^{1} \rightarrow^{\pi} \mathbb{A}^{1}$ is the total family. Now $E_{1}$ is contained in a smooth surface of degree $-g+1$ (in fact, $E_{1} \subset D_{1} \cup L$ and $D_{1} \cup L$ is contained in a smooth surface of degree $-g+1$ by $\left[22,2.6(\right.$ a) $]$ ) with equation $s_{1}$. Thus we can find a section $s_{t}$ extending $s_{1}$ with $s_{0}=h$ and we let $S_{t}$ be the corresponding family of surfaces.

Now consider the family $\left.C_{t}=L \cup\left(S_{t} \cap L_{0}^{\{2\}}\right) \cup L_{t}\right)$. For $t \in U, U \subset \mathbb{A}^{1}$ a nonempty open subset, $C_{t}$ is the disjoint union of $L_{t}$ and $Z_{t} \cup_{2 P} L$, where $Z_{t}$ is a double line of genus $g$. Since $I_{Z_{t}}=\left((x, y)^{2}, s_{t}\right)$ and $L_{t} \subset Q \cap S_{t}$, we obtain the containment

$$
\left((x+w t, y+z t)(x, y)^{2},(x, y) q, s_{t}\right) \subset I_{Z_{t}} \cap I_{L_{t}}
$$

and conclude that $\lim _{t \rightarrow 0} Z_{t} \cup L_{t} \subset W_{0}$ in taking the limit as $t \rightarrow 0$. It follows that $\lim _{t \rightarrow 0} C_{t} \subset W_{0} \cup L$ in the full Hilbert scheme. Since the Hilbert polynomials agree, $W_{0}=\lim C_{t}$. 


\section{The Hilbert Schemes $H_{4, g}$}

In this section we prove the main results of the paper. The first of these is Theorem 6.2 that describes the irreducible components of the Hilbert schemes $H_{4, g}$. The second is the fact 6.4 that $H_{4, g}$ is connected. The cases when $g \geqslant 0$ are well known and described in the introduction. We begin with the case $g=-1$, since it has a somewhat different statement due to the existence of more reduced curves.

PROPOSITION 6.1. The Hilbert scheme $H_{4,-1}$ is connected and has irreducible components as shown in Table II.

Proof. If a curve $C \in H_{4,-1}$ is not extremal, then its spectrum is necessarily $\left\{0^{2}, 1^{2}\right\}$, in which case $C$ is in one of the families $H_{2}$ or $H_{3}$ of Proposition 4.2. These families have general members as described in Remark 4.4 and meet because both contain thick 4-lines. Finally, $H_{2}$ meets the family of extremal curves by [23] or [14].

THEOREM 6.2. The Hilbert schemes $H_{4, g}$ have irreducible components as shown in Table III for $g \leqslant-2$.

Proof. In the table $G_{j}$ denotes the closure in the Hilbert scheme $H_{4, g}$ of the set of curves described in the corresponding row. The outline of the proof is as follows. First we show the families listed in the table are irreducible of the stated dimension. Then we show there is no inclusion relation among them. Finally, we prove every curve of degree 4 and genus $g \leqslant-2$ belongs to one of these families. The restrictions given in the table are necessary to ensure that a given family exists and is not contained in another family of the list.

The family $G_{1}$ consists of extremal curves, and is an irreducible component of the Hilbert scheme of dimension $15-2 g$ by $[18,4.3] . G_{2}, G_{3}$ and $G_{4}$ are the closures of the families $\mathrm{H}_{2}, \mathrm{H}_{3}$ and $H_{n(g)}$ of Proposition 4.3, and are therefore irreducible of the stated dimension. Note that $G_{2}$ contains the subextremal curves and $G_{4}$ consists of thick 4-lines. Since any double conic lies on a double plane (if the double conic $C$ has support $Y$, then $C \subset Y^{(2)}$, i.e. $I_{Y}^{2} \subset I_{C}$ ), the closure $G_{5}$ of the family of double conics of genus $g$ is irreducible of dimension $13-2 g$ because the family of such curves in a fixed double plane $2 H$ is irreducible of dimension $10-2 g$ by [14, 2.1 and 4.3$]$. The

Table II. Irreducible components of the Hilbert scheme $H_{4,-1}$

\begin{tabular}{lll}
\hline Label & General curve & Dimension \\
\hline$H_{1}$ & extermal curve & 17 \\
$H_{2}$ & disjoint union of two conics & 16 \\
$H_{3}$ & disjoint union of line and & 16 \\
& twisted cubic & \\
\hline
\end{tabular}


Table III. Irreducible components of the Hilbert scheme $H_{4, g}$ for $g \leqslant-2$

\begin{tabular}{|c|c|c|c|}
\hline Label & General curve & Dimension & Restrictions \\
\hline$G_{1}$ & $\begin{array}{l}D \cup Z \\
D \text { smooth conic } \\
g(Z)=g-3, \text { length }(D \cap Z)=4\end{array}$ & $15-2 g$ & none \\
\hline$G_{2}$ & $\begin{array}{l}L_{1} \cup_{2 P} Z \cup_{2 Q} L_{2} \\
L_{1} \cap L_{2}=\emptyset \\
g(Z)=g-2\end{array}$ & $13-2 g$ & none \\
\hline$G_{3}$ & $\begin{array}{l}D \cup_{2 P} Z \\
D \text { smooth conic } \\
g(Z)=g-1\end{array}$ & $13-2 g$ & none \\
\hline$G_{4}$ & general thick 4-line & $9-3 g$ & $g \leqslant-3$ \\
\hline$G_{5}$ & double conic & $13-3 g$ & none \\
\hline$G_{6}$ & $\begin{array}{l}Z \cup_{2 P} L_{1} \dot{\cup} L_{2} \\
g(Z)=g\end{array}$ & $11-2 g$ & $g \leqslant-3$ \\
\hline$G_{7, a}$ & $\begin{array}{l}W \text { quasiprimitive } 3 \text {-line } \\
\quad \text { of type }(a,-3 a-g)\end{array}$ & $11-2 g-a$ & $\begin{array}{l}g \leqslant-3 \\
0<a \leqslant \frac{-g}{3}\end{array}$ \\
\hline$G_{8, a}$ & $\begin{array}{l}W \cup_{2 P} L \\
W \text { quasiprimitive } 3 \text {-line } \\
\quad \text { of type }(a,-1-3 a-g)\end{array}$ & $10-2 g-a$ & $\begin{array}{l}g \leqslant-6 \\
0<a \leqslant \frac{-g-1}{3}\end{array}$ \\
\hline$G_{9, a}$ & $\begin{array}{l}W \cup \dot{L} L \\
W \text { quasiprimitve } 3 \text {-line } \\
\quad \text { of type }(a,-3-3 a-g)\end{array}$ & $8-2 g-a$ & $\begin{array}{l}g \leqslant-6 \\
0<a \leqslant \frac{-g-3}{3}\end{array}$ \\
\hline$G_{10, m}$ & $\begin{array}{l}D_{1} \dot{\cup} D_{2} \\
\operatorname{deg}\left(D_{1}\right)=2, g\left(D_{1}\right)=-m \\
\operatorname{deg}\left(D_{2}\right)=2, g\left(D_{2}\right)=g+m+1\end{array}$ & $\mathrm{~d}(-\mathrm{m})+\mathrm{d}(\mathrm{g}+\mathrm{m}+1)$ & $\begin{array}{l}0 \leqslant m \leqslant \frac{-q-1}{2} \\
g \leqslant-9\end{array}$ \\
\hline$G_{11, a, b}$ & $\begin{array}{l}\text { Quasiprimitive 4-line } \\
\quad \text { of type }(a, b,-6 a-b-g-3)\end{array}$ & $\begin{array}{l}7-2 g-3 a \\
\quad=9 a+2 b+2 c+13\end{array}$ & $\begin{array}{l}g \leqslant-9 \\
0<a \leqslant \frac{-g-3}{6} \\
0 \leqslant b \leqslant \frac{-6 a-g-3}{2}\end{array}$ \\
\hline
\end{tabular}

Notation: The letter $L$ denotes a line, the letter $Z$ denotes a double line, and $\dot{U}$ indicates a disjoint union. $L \cup_{n P} C$ stands for the union of a line $L$ and a curve $C$ which intersect along the divisor $n P$ on $L$. In the tenth entry we have $d(-m)=\operatorname{dim} H_{2,-m}$ equals $5+2 m$ if $m>1$, and 8 if $m=0$ or $m=1$.

families $G_{7, a}$ and $G_{8, a}$ are the closures of $F_{1, a}$ and $F_{3, a}$ from Proposition 5.2 and are therefore irreducible of the stated dimension. The irreducibility of $G_{11, a, b}$ is proven in Proposition 2.3

Finally, families $G_{6}, G_{9, a}$ and $G_{10, m}$ are irreducible components of $H_{4, g}$ because the curves defining them are disjoint unions of curves that are general in their respective Hilbert schemes. The dimensions of these families can be computed out of $[20,1.6$, 3.4, 3.5].

This shows all families in the statement are irreducible, and we now prove there are no inclusion relation among them. We have just seen that $G_{1}, G_{6}, G_{9, a}$ and 
$G_{10, m}$ are irreducible components of $H_{4, g}$, so certainly none of them can be contained in any other family of the list.

The families $G_{2}, G_{3}$ and $G_{5}$ could only be contained in $G_{1}$ or $G_{4}$ by reason of dimension. However, none of them is contained in $G_{1}$ by semicontinuity, and none of them is contained in $G_{4}$ because curves in $G_{4}$ are supported on a single line.

In the table, the only families of dimension larger than that of $G_{4}$ consist of curves $C$ with $h^{0} \mathcal{I}_{C}(2) \neq 0$. These cannot specialize to a general thick 4-line $T$ because $h^{0} \mathcal{I}_{T}(2)=0$ for $g \leqslant-3$ by Proposition 4.3. Thus $G_{4}$ is not contained in any of the other families. Note however that $G_{3}$ contains all thick 4-lines when $g=-2$.

We now treat the case of $G_{7, a}$ and $G_{8, a}$. The general curves in $G_{7, a}$ and $G_{8, a}$ are not supported on a line, so they can't be contained in $G_{4}$ or $G_{11, a, b}$. $G_{7, a}$ and $G_{8, a}$ are not contained in $G_{1}, G_{2} G_{3}$ or $G_{5}$ by semicontinuity - curves in the latter families have larger speciality by Proposition 4.3.

$G_{7, a}$ and $G_{8, a}$ are not contained in $G_{6}$ or $G_{10, m}$ because when two lines collapse the resulting double line has genus $\geqslant-1$, hence $a \leqslant 0$ contradicting the restriction imposed.

If $G_{7, a^{\prime}} \subset G_{9, a}$, then $a^{\prime} \leqslant a$ by Remark 2.5 , which in turn implies that $\operatorname{dim} G_{7, a^{\prime}}=$ $11-2 g-a^{\prime}>8-2 g-a=\operatorname{dim} G_{9, a}$, a contradiction. Similarly $G_{8, a^{\prime}} \not \subset G_{9, a}$.

It remains to show that neither $G_{7, a}$ and $G_{8, a}$ contains the other. There can be no containment $G_{7, a^{\prime}} \subset G_{8, a}$, because then $a^{\prime} \leqslant a$ by Remark 2.5 and hence $\operatorname{dim} G_{8, a}=10-2 g-a<11-2 g-a^{\prime}=\operatorname{dim} G_{7, a}$, a contradiction. Now suppose that $G_{8, a^{\prime}} \subset G_{7, a}$. Remark 2.5 tells us again that $a^{\prime} \leqslant a$, and since $\operatorname{dim} G_{8, a^{\prime}}<$ $\operatorname{dim} G_{7, a}$ we conclude that $a^{\prime}=a$. In particular, the limit of the underlying family of double lines $Z$ has no embedded points. This is not possible because the limit double line meets $L$ in one point while the general member meets $L$ in a double point.

Finally, $G_{11, a, b}$ cannot be contained in any of the families $G_{j}$ with $j \leqslant 10$ by semicontinuity: indeed, since $a>0$, every 4-line $C$ in $G_{11, a, b}$ satisfies $h^{1} \mathcal{O}_{C}(-2)=1$, while for any other curve $D \in H_{4, g}$ we have $h^{1} \mathcal{O}_{D}(-2) \geqslant 2$. On the other hand, there are no containments among the families $G_{11, a, b}$ : if $G_{11, a, b} \subset G_{11, a^{\prime}, b^{\prime}}$, then by Remark 2.5 we would have $a \leqslant a^{\prime}$, while

$$
7-2 g-3 a^{\prime}=\operatorname{dim} G_{11, a^{\prime}, b^{\prime}}>\operatorname{dim} G_{11, a, b}=7-2 g-3 a
$$

would yield $a^{\prime}<a$, a contradiction.

To finish the proof, we still have to show our families cover the Hilbert scheme. Let $C \in H_{4, g}$ have support $B=C_{\text {red }}$.

Case 1. $\operatorname{deg} B=4$

Here $C=B$ is reduced, and all reduced curves of degree 4 satisfy $g \geqslant-1$ with the following two exceptions: either (a) $C$ is the disjoint union of a conic (possibly degenerate) and two lines, when $g=-2$ and $C \in G_{10,0}$ or (b) $C$ is the disjoint union of four lines, $g=-3$ and $C \in G_{10,1}$. 
Case 2. $\operatorname{deg} B=3$

In this case $C=Z \cup D$, where $Z$ is a double line with support $L$ and $D$ is a reduced curve of degree 2. In particular, $B=L \cup D$.

First suppose that $D$ is planar and let $l=\operatorname{length}(D \cap L)$. If $l=0$, then $C \in G_{10,0}$. If $l=1$, then $g(B)=0$ and $C$ belongs to one of the families $G_{2}, G_{3}$ or $G_{4}$ by Proposition 4.3. If $l=2$, then $B$ is planar and hence $C$ is extremal by [21,2.2].

The other possibility is that $D=L_{1} \cup L_{2}$ is a disjoint union of lines. If $D$ does not meet $Z$, then $C \in G_{10,1}$. If $Z$ meets $L_{1}$ but not $L_{2}$, then $Z \cup L_{1}$ is a specialization of a double line meeting $L_{1}$ in a double point by [20,3.2], so $C$ lies in $G_{6}$. If $Z$ meets both $L_{1}$ and $L_{2}$, then $g(B)=0$ and $C$ again belongs to one of the families $G_{2}, G_{3}$ or $G_{4}$ by Proposition 4.3 .

Case 3. $\operatorname{deg} B=2$

If $B$ is a smooth conic, then $C$ belongs to $G_{5}$, so we suppose that $B=L \cup L^{\prime}$ is a union of two lines. If $C$ is a union of double lines, then either (a) the lines are disjoint and $C \in G_{10, m}$ for $m=-\max \left\{g\left(Z_{1}\right), g\left(Z_{2}\right)\right\}$ or (b) the lines meet and hence $C$ is contained in a double plane; in this case $C \in G_{5}$ by [14, 8.1 and 8.2].

The remaining possibility is that $C=W \cup L$, where $L$ is a line and $W$ is a triple line which is quasi-primitive because $g(W) \leqslant-1$ (the only thick triple line has genus 0 ). If $W$ has type $(a, b)$, let $Z$ be the underlying double line and set $l=$ length $(W \cap L)$.

If $l=3$, then necessarily length $(Z \cap L)=2$. If $a=-1$, then $Z \cup L$ is planar and $C$ is extremal. If $a=0$, then $Z \cup L \in H_{3,0}$ and $C$ belongs to one of the families $G_{2}, G_{3}$ or $G_{4}$ by Proposition 4.3. If $a>0$, then $C$ belongs to $G_{7, a}$.

Suppose $l=2$. If length $(Z \cap L)=2$ and $a=-1$ or 0 , we argue as in the case $l=3$ above. If length $(Z \cap L)=2$ and $a>0$, then $C \in G_{7, a}$ by Proposition 5.4. Thus we may assume $Z \cap L=P$ a reduced point. If $a=-1$, then $Z \cup L \in H_{3,0}$ and $C \in G_{2} \cup G_{3}$ by Proposition 4.3, while if $a=0$, then $C \in G_{6}$ by Remark 5.6. If $a>0$, then $C \in G_{8, a}$.

Suppose $l=1$. Then either (a) $a>0$ so that $C \in G_{8, a}$ by Proposition. 5.4 (b) $a=0$ and $C \in G_{6}$ by Proposition 5.4 and Remark 5.6 or (c) $a=-1, Z \cup L \in H_{3,0}$ and $C \in G_{2} \cup G_{3}$ by Proposition 4.3.

If $l=0$, then $C=W \cup L$ with $W$ a quasi-primitive 3-line of type $(a,-3-3 a-g)$. Now either (a) $a=-1, W$ is extremal and $C \in G_{6}$ by [20,3.2] or (b) $a=0$ and $C \in G_{5,0}$ by [20,3.3] or (c) $a>0$ and $C \in G_{9, a}$.

Case 4. $\operatorname{deg} B=1$

If $C$ is thick, then $C \in G_{4}$. If $C$ is a quasiprimitive 4-line, then $C$ has type $(a, b, c)$ for some integers $a \geqslant-1$ and $c \geqslant b \geqslant 0$. If $a=-1$, then the underlying double line $Z$ is planar and $C$ lies in a double plane, hence $C \in G_{5}$ by [14, 8.1 and 8.2]. If $a=0$, then $C$ is in $G_{10, m}$ for $m=-1-b$ by Proposition 3.3. Finally, if $a>0$, then $C \in G_{11, a, b}$.

EXAMPLE 6.3. As the restrictions in Table III imply, some of the components do not show up if the genus $g$ is not small enough. The five irreducible components of $H_{4,-2}$ appear in Table IV: 
Table IV. Irreducible components of $H_{4,-2}$

\begin{tabular}{lll}
\hline Label & Description & Dimension \\
\hline$G_{1}$ & $\begin{array}{l}\text { Conic meeting a double line } \\
\text { in a scheme of length 4 } \\
L_{1} \cup_{2 P} Z \cup_{2 Q} L_{2} L_{i} \text { lines, } Z \text { a } \\
\text { double line of genus - }\end{array}$ & 19 \\
$\begin{array}{l}\text { Conic meeting a double line } \\
\text { in a double point }\end{array}$ & 17 \\
$G_{3}$ & $\begin{array}{l}\text { Double conic } \\
\text { Disjoint union of a conic and } \\
G_{5}\end{array}$ & 17 \\
$G_{10,0}$ & two lines & 17 \\
& & 16 \\
\hline
\end{tabular}

We can now prove $H_{4, g}$ is connected:

THEOREM 6.4. The Hilbert scheme $H_{4, g}$ is connected if nonempty.

Proof. We may assume $g \leqslant-2$, and it suffices to show that all the irreducible components can be connected to the component $G_{1}$ of extremal curves. The families $G_{2}$ and $G_{3}$ meet the family $G_{4}$ of thick 4-lines by Proposition 4.3, and $G_{2}$ meets $G_{1}$ by [1, 14], or [23]. In particular, thick 4-lines belong to the connected component of extremal curves, and it is enough to show that all other components can be connected to either $G_{1}$ or $G_{4}$. $G_{5}$ meets $G_{1}$ by [14, 5.1 and 8.2]. That $G_{6}$ meets $G_{1}$ follows immediately from [11,2.1] and [20]. $G_{7, a}$ meets $G_{1}$ by Corollary 5.5 and $G_{8, a}$ meets $G_{1}$ by Proposition 5.4 and $[11,2.5]$. One can connect $G_{9, a}$ to $G_{1}$ by applying $[20,3.8]$ and $[11,2.1]$. The families $G_{11, a, b}$ meet $G_{4}$ by Proposition 2.4. Applying Proposition 3.3, we see $G_{10, m}$ contains $G_{11,0, m-1}$ for $m>0$ and, hence, meets $G_{4}$ as well. Lastly, we consider $G_{10,0}$. By definition this family contains curves $C=Z \cup L_{1} \cup_{P} L_{2}$ where $Z$ has degree two and $L_{i}$ are meeting lines. Since $Z$ is extremal, $C$ specializes to an extremal curve $E \in G_{1}$ by [11,2.1 and 2.5].

As an application of our results, we can now give a counterexample to a conjecture of Aït-Amrane and Perrin [2]. The conjecture regards the following question, which has been a recurring theme of this paper:

QUESTION 6.5. Let $X$ and $X_{0}$ be two irreducible families of curves in $H_{d, g}$ having constant cohomology. Under what conditions do we have a nonempty intersection $\bar{X} \cap X_{0} \neq \emptyset$ in $H_{d, g}$ ?

We have been lucky in that whenever we suspected the existence of such a deformation, we could actually prove it. In general, this question is difficult. Semicontinuity [8, III, Section 12] gives a necessary condition: If $\tau^{i}(n)=h^{i}\left(\mathbb{P}^{3}, \mathcal{I}_{C}(n)\right)$ for all $C \in X$ and $\tau_{0}^{i}(n)=h^{i}\left(\mathbb{P}^{3}, \mathcal{I}_{C_{0}}(n)\right)$ for all $C_{0} \in X_{0}$, then whenever $\bar{X} \cap X_{0} \neq \emptyset$ we must 
have $\tau^{i}(n) \leqslant \tau_{0}^{i}(n)$ for all $i$ and $n$ (we write $\tau \leqslant \tau_{0}$ for short). This condition is not sufficient, even when $X$ (resp. $X_{0}$ ) is an irreducible component of the Hilbert scheme $H_{\tau}$ (resp. $H_{\tau_{0}}$ ) of curves with fixed cohomology. This has been shown by a recent example of Ait-Amrane and Perrin [2].

A more subtle necessary condition is afforded by the Rao modules of the curves. Let $A$ be a discrete valuation ring with fraction field $K$ and residue field $k$ and let $\mathcal{C} \subset \mathbb{P}_{A}^{3}$ be a family of locally Cohen-Macaulay curves over $A$. Then the Rao module $M_{C_{K}}$ of the generic curve is a flat deformation of a subquotient $M$ of the Rao module $M_{C_{k}}$ of the special curve ([13, Proposition 5.9], [1, $\left.\S 4.2 .2\right]$, [2, Proposition 13]). This means that there are submodules $M_{1} \subset J \subset M_{C_{k}}$ such that $M=J / M_{1}$ and $M$ is a flat deformation of $M_{C_{K}}$. Moreover,

$$
\begin{aligned}
& \operatorname{dim}\left(M_{1}\right)_{n}=h^{0} \mathcal{I}_{C_{k}}(n)-h^{0} \mathcal{I}_{C_{K}}(n), \\
& \operatorname{dim}\left(M_{C_{k}} / J\right)_{n}=h^{2} \mathcal{I}_{C_{k}}(n)-h^{2} \mathcal{I}_{C_{K}}(n) .
\end{aligned}
$$

In view of this result, the following conjecture of Ait-Amrane and Perrin is natural:

CONJECTURE 6.6 ([2], Conjecture 14). Let $X$ and $X_{0}$ be irreducible components of $H_{\tau}$ and $H_{\tau_{0}}$ respectively. Suppose that

(1) $\tau \leqslant \tau_{0}$

(2) The Rao module of the generic curve $C_{\xi}$ of $X$ is a flat deformation of a subquotient of the Rao module of a curve $C_{0}$ in $X_{0}$ and that the numerical conditions (13) and (14) hold.

Then $\bar{X} \cap X_{0} \neq \emptyset$ in $H_{d, g}$.

As it turns out, the conditions of the conjecture are still insufficient, as we note in the following example.

EXAMPLE 6.7. For $g \leqslant-3$, let $X \subset H_{4, g}$ denote the irreducible family $H_{n(g)}$ from Proposition 4.3. The closure $\bar{X}$ is precisely the family of thick 4-lines. Let $X_{0}=G_{1}$ denote irreducible family consisting of extremal curves. We claim that $\bar{X} \cap X_{0}=\emptyset$. Indeed, an extremal curve cannot specialize to a thick 4-line because this would violate semicontinuity, while a thick 4-line has everywhere embedding dimension 3, and so cannot specialize to an extremal curve that has generic embedding dimension 2 [18]. On the other hand, we will now show that the conditions of the conjecture hold.

First we compare the Rao modules. Let $C$ be a general thick 4-line with support $L$ and set $S=S_{L}$. Proposition 4.3 shows that $M_{C} \cong S /(a, b, c)(-g-1)$ where $a, b, c$ are general forms of degree $-g+1$ in $S$. Choose a linear form $l \in S$ so that $(a, l)$ is a regular sequence and a form $f \in(b, c)$ of degree $-g+2$ so that $(a, f)$ is a regular sequence. We consider the extremal Koszul module $M=S /(a, l f)$. Since the multiplication $S /(a) \stackrel{\cdot}{\rightarrow} S /(a)$ is injective and the image of the submodule $(\bar{f})$ is 
$(\overline{l f})$, we see that the submodule $J=\bar{l} M$ is isomorphic to $S /(a, f)$. Since $f \in(b, c)$ by choice, $S /(a, b, c)$ is a quotient of $J$ by $M_{1}=(l b, l c)$. If $E$ is an extremal curve corresponding to $M$, then $\operatorname{deg} E=4, p_{a}(E)=g$ and we have just shown that $M_{C}$ is a subquotient of $M_{E}$. It is clear that $\operatorname{dim}(M / J)_{n}=1$ for $g \leqslant n \leqslant 0$ and zero otherwise; this is seen to be precisely $h^{2} \mathcal{I}_{E}(n)-h^{2} \mathcal{I}_{C}(n)$ in comparing the spectra of these curves. Finally, since the Euler characteristics of $\mathcal{I}_{E}$ and $\mathcal{I}_{C}$ are the same, the exact sequences relating the Rao modules shows that $\operatorname{dim}\left(M_{1}\right)_{n}=h^{0} \mathcal{I}_{E}(n)-h^{0} \mathcal{I}_{C}(n)$. In particular, the semicontinuity conditions are immediate.

\section{Acknowledgement}

We would like to thank the referee for a very careful reading and helpful comments, especially in Section 3.

\section{References}

1. Aït-Amrane, S.: Sur le schéma de Hilbert des courbes gauches de degré $d$ et genre $g=\frac{1}{2}(d-3)(d-4)$, Thesis, Univ. de Paris Sud, Centre d'Orsay, 1998.

2. Aït-Amrane, S. and Perrin, D.: Un contre-exemple sur les familles de courbes gauches, Comm. Algebra 28 (2000), 6003-6015 (Special issue in honor of Robin Hartshorne).

3. Banica, C. and Forster, O.: Multiplicity structures on space curves, In: Lefschetz Centennial Conference, Contemporary Math. 58, Amer. Math. Soc., Providence, 1986, pp. 47-64.

4. Ballico, E., Bolondi, G. and Migliore, J.: The Lazarsfeld-Rao problem for liaison classes of two-codimensional subschemes of $\mathbb{P}^{n}$, Amer. J. Math. 113 (1991), 117-128.

5. Buchsbaum, D. and Eisenbud, D.: What makes a complex exact? J. Algebra 25 (1973), 259-268.

6. Ellingsrud, G.: Sur le schéma de Hilbert des variétés de codimension 2 dans $\mathbb{P}^{e}$ à cône de Cohen-Macaulay, Ann. Sci. École Norm. Sup. (4) 8 (1975), 423-431.

7. Gruson, L. and Peskine, C.: Genre des courbes de l'espace projectif, In: Algebraic Geometry, Tromsø 1977, Lecture Notes in Math. 687, Springer-Verlag, New York, 1978, pp. 31-59.

8. Hartshorne, R.: Algebraic Geometry, GTM 52, Springer-Verlag, Berlin, 1977.

9. Hartshorne, R.: Generalized divisors on Gorenstein schemes, K-Theory 8 (1994), 287-339.

10. Hartshorne, R.: The genus of space curves, Annali Univ. Ferrara, Sez. VII - Sci. Mat. 40 (1994), 207-223.

11. Hartshorne, R.: On the connectedness of the Hilbert scheme of curves in $\mathrm{P}^{3}$, Comm. Algebra 28 (2000), 6059-6077.

12. Hartshorne, R.: Questions of Connectedness of the Hilbert Scheme of Curves in $\mathrm{P}^{3}$, Preprint math.AG/0104265, To appear in the Proc. Abhyankar Conference, Purdue Univ. 2000, Springer-Verlag, New York.

13. Hartshorne, R., Martin-Deschamps, M. and Perrin, D.: Triades et familles de courbes gauches, Math. Ann. 315 (1999), 397-468.

14. Hartshorne, R. and Schlesinger, E.: Curves in the double plane, Comm. Algebra 28 (2000), $5655-5676$.

15. Jouanolou, J. P.: Théorémes de Bertini et applications, Progr. in Math. 42, Birkhäuser, Boston, 1983.

16. Martin-Deschamps, M. and Perrin, D.: Sur la classification des courbes gauches, Astérisque (1990), 184-185. 
17. Martin-Deschamps, M. and Perrin, D.: Sur les bornes du module de Rao, C.R. Acad. Sci. Paris, Sér. I 137 (1993), 1159-1162.

18. Martin-Deschamps, M. and Perrin, D.: Le schéma de Hilbert des Courbes Gauches localement Cohen-Macaulay n'est (presque) jamais réduit, Ann. Sci. École Norm. Sup. (4) 29 (1996), 757-785.

19. Migliore J.: Geometric invariants for liaison of space curves, J. Algebra 99 (1986), $548-572$.

20. Nollet, S.: The Hilbert schemes of degree three curves, Ann. Sci. École Norm. Sup. (4) 30 (1997), 367-384.

21. Nollet, S.: Subextremal curves, Manuscripta Math. 94 (1997), 303-317.

22. Nollet, S.: Bounds on multisecant lines, Collect. Math. 49 (1998), 447-463.

23. Nollet, S.: A remark on connectedness in Hilbert schemes, Comm. Algebra 28 (2000), 5745-5747.

24. Nollet, S. and Schlesinger, E.: Curves on a double surface, to appear in Collect. Math.

25. Perrin, Daniel.: Un pas vers la connexité du schéma de Hilbert: les courbes de Koszul sont dans la composante des extrémales, Collect. Math. 52 (2001), 295-319.

26. Sabadini, I.: On the Hilbert schemes of curves of degree $d$ and genus $\left(\begin{array}{c}d-3 \\ 2\end{array}\right)-1$, to appear in Le Matematiche, 2002, Special issue in honor of Silvio Greco.

27. Schlesinger, E.: The spectrum of projective curves, Thesis, University of California at Berkeley 1996.

28. Schlesinger, E.: A speciality theorem for Cohen-Macaulay space curves, Trans. Amer. Math. Soc. 351 (1999), 2731-2743. 